\title{
TRANSIENCE AND THERMODYNAMIC FORMALISM FOR INFINITELY BRANCHED INTERVAL MAPS
}

\author{
HENK BRUIN AND MIKE TODD
}

\begin{abstract}
We study a one-parameter family of countably piecewise linear interval maps, which, although Markov, fail the 'large image property'. This leads to conservative as well as dissipative behaviour for different maps in the family with respect to Lebesgue. We investigate the transition between these two types, and study the associated thermodynamic formalism, describing in detail the second order phase transitions (i.e., the pressure function is $C^{1}$ but not $C^{2}$ at the phase transition) that occur in transition to dissipativity. We also study the various natural definitions of pressure which arise here, computing these using elementary recurrence relations.
\end{abstract}

\section{INTRODUCTION}

The aim of this paper is to understand thermodynamic formalism of a simple class of infinitely branched uniformly expanding interval maps with suboptimal mixing properties. Given $\lambda \in(0,1)$, our system is a countably piecewise linear interval map $F_{\lambda}:(0,1] \rightarrow(0,1]$, defined as

$$
F_{\lambda}(x):= \begin{cases}\frac{x-\lambda}{1-\lambda} & \text { if } x \in W_{1}, \\ \frac{x-\lambda^{n}}{\lambda(1-\lambda)} & \text { if } x \in W_{n}, \quad n \geqslant 2,\end{cases}
$$

for the intervals $W_{n}:=\left(\lambda^{n}, \lambda^{n-1}\right]$, which form a Markov partition.

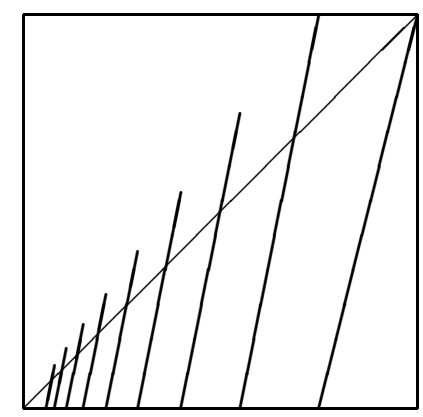

$$
\ldots W_{4} W_{3} \quad W_{2} \quad W_{1}
$$

This map was proposed by van Strien to Stratmann as a model for an induced map of Fibonacci unimodal map. Stratmann \& Vogt SV] computed the Hausdorff dimension of points that converge to 0 under iteration of $F_{\lambda}$ (and in fact this set has full Lebesgue

Date: Version of November 25, 2019.

2000 Mathematics Subject Classification. 37E05 37D35 60J10 37D25 37A10 .

Key words and phrases. Transience, thermodynamic formalism, interval maps, Markov chains, equilibrium states, non-uniform hyperbolicity.

The hospitality of the Mittag-Leffler Institute on Djursholm, Stockholm (2010 Spring programme on Dynamics and PDEs) is gratefully acknowledged. MT was partially supported by NSF grants DMS 0606343 and DMS 0908093. He would also like to thank A Hoffman, N Dobbs and G Iommi for useful conversations in the early stages of this project. 
measure for $\lambda>\frac{1}{2}$ ), which has a bearing on the existence and nature of wild attractors in interval dynamics, BKNS]. Bruin showed (unpublished), that the map $F_{\lambda}$ is indeed an induced map of a countably piecewise linear unimodal map, but we intend to come back to this issue in a forthcoming paper. The goal of this paper is to investigate the thermodynamic properties of $\left((0,1], F_{\lambda}\right)$ which is of interest in its own right. A hint that piecewise expanding maps with countably many pieces can be Lebesgue dissipative was made early on by Lasota \& Yorke [LY, page 487]. A large part of the current theory of Markov maps with infinitely many branches relies on a "large image property", which $F_{\lambda}$ does not satisfy. In contrast, the distinction between dissipative (transient) and conservative (recurrent) behaviour leads to second order phase transition (see below) at $t=t_{0}=\frac{-\log 2}{\log \lambda}$ for the 'geometric' potential $\Phi_{t}=-t \log \left|F_{\lambda}^{\prime}\right|$ (which is assumed to be the appropriate one-sided derivative at each discontinuity point $\lambda^{n}$ ).

Our first main theorem describes the existence of $(\phi-p)$-conformal reference measures, see Definition 1 for their precise definition. Let

$$
P_{\text {Conf }}(\phi):=\inf \{p \in \mathbb{R}: \text { there exists a }(\phi-p) \text {-conformal measure }\} .
$$

When the potential is $\Phi_{t}$, for brevity we will also call a $\left(\Phi_{t}-p\right)$-conformal measure a $(t, p)$-conformal measure.

Letting $\psi(t):=\frac{(1-\lambda)^{t}}{1-\lambda^{t}}$, we have the following expression for $P_{\mathrm{Conf}}\left(\Phi_{t}\right)$.

Theorem A. Given $\lambda \in(0,1)$ and $t \in \mathbb{R}$,

$$
P_{\text {Conf }}\left(\Phi_{t}\right)= \begin{cases}\log \psi(t) & \text { if } \lambda^{t} \leqslant \frac{1}{2} ; \\ \log \left[4 \lambda^{t}(1-\lambda)^{t}\right] & \text { if } \lambda^{t} \geqslant \frac{1}{2} .\end{cases}
$$

If $p=P_{\mathrm{Conf}}\left(\phi_{t}\right)$ then there exists a $(t, p)$-conformal measure $m_{t, p}$. This measure is

$$
\begin{cases}\text { conservative } & \text { if } \lambda^{t} \leqslant \frac{1}{2}, \\ \text { dissipative } & \text { if } \lambda^{t}>\frac{1}{2} .\end{cases}
$$

If $p \neq P_{\mathrm{Conf}}\left(\Phi_{t}\right)$, then $m_{t, p}$ is dissipative.

As we are mostly interested in the case $p=P_{\text {Conf }}\left(\Phi_{t}\right)$, we will often abbreviate $m_{t}=$ $m_{t, p}$ when $p=P_{\text {Conf }}\left(\Phi_{t}\right)$. We define the pressure as

$$
P\left(\Phi_{t}\right):=\sup \left\{h(\mu)+\int \Phi_{t} d \mu: \mu \in \mathcal{M},-\int \Phi_{t} d \mu<\infty\right\},
$$

where the supremum is taken over the set $\mathcal{M}$ of $F$-invariant probability measures. A measure $\mu \in \mathcal{M}$ such that $h(\mu)+\int \Phi_{t} d \mu=P\left(\Phi_{t}\right)$ is called an equilibrium state for $\Phi_{t}$.

The behaviour of the function $t \mapsto P\left(\Phi_{t}\right)$ is important for understanding the statistical properties of the system. In the classical hyperbolic case, this function is real analytic [R2]. We say that the pressure $t \mapsto P\left(\Phi_{t}\right)$ has a $k$-th order phase transition at $t_{0}$ if this function is $C^{k-1}$, but not $C^{k}$ at $t_{0}$. In the following theorem, we see that our pressure function has a second order phase transition at $t_{0}=\frac{-\log 2}{\log \lambda}$. 
Theorem B. Given $\lambda \in(0,1)$ and $t \in \mathbb{R}$,

$$
P\left(\Phi_{t}\right)= \begin{cases}\log \psi(t) & \text { if } \lambda^{t} \leqslant \frac{1}{2} \\ \log \left[4 \lambda^{t}(1-\lambda)^{t}\right] & \text { if } \lambda^{t} \geqslant \frac{1}{2},\end{cases}
$$

so there is a second order phase transition at $t_{0}=\frac{-\log 2}{\log \lambda}$. Moreover, there is an equilibrium state $\mu_{t}$ for $\Phi_{t}$ if $\lambda^{t}<1 / 2$. If such an equilibrium state exists, it is unique and is absolutely continuous w.r.t. $m_{t}$. There is no equilibrium state for $\Phi_{t}$ when $\lambda^{t} \geqslant 1 / 2$; in particular, there is no measure of maximal entropy.

For $t=0$ we arrive at the topological entropy $h_{\text {top }}\left(F_{\lambda}\right)=\log 4$ for all $\lambda \in(0,1)$. It may be a bit surprising that a transitive map with countably many (expanding) branches has finite entropy, but this phenomenon has been observed before, e.g. [Rt, $\mathrm{MR}, \mathrm{BS}$. The non-existence of a measure of maximal entropy goes back to Gurevich's paper G2, which basically says that the only measure of maximal entropy is given by a normalised eigenvector (with eigenvalue 1) for the infinite transition matrix associated with the Markov shift. In our terminology, this is the matrix $A^{t}$ with $t=0$ (see (13)), and the required eigenvector is indeed non-existent because conformal measure $m_{t, p}$ is dissipative for $t=0, p=\log 4$. Based on work by Gurevich [G2] and Salama [Sl], Ruette [Rt] presents examples of $C^{r}$ interval maps with infinitely many branches, finite topological entropy but no measure of maximal entropy.

For the case when the dynamical system $(X, f)$ is a countable Markov shift, and $\phi: X \rightarrow \mathbb{R}$ is a sufficiently smooth potential, Sarig [S2] defined recurrence, and its converse, transience, in terms of local partition functions (see Section 6). If the system is recurrent, then he gave a further condition on such functions under which the system is positive recurrent; the converse of which is null recurrent. He proved that in this context, recurrence is equivalent to the existence of a conservative (see Definition 2 below) $(\phi-P(\phi))$-conformal measure $m$ (see Theorem 4). Moreover, if the system is recurrent, it is positive recurrent if there exists an $f$-invariant probability measure $\mu \ll m$, and null recurrent otherwise. In [T], it was shown that it is reasonable and useful, in order to apply these ideas beyond the realm of shift spaces, to take the conditions on the existence (or non-existence) of such conformal and invariant measures as the definition of the two kinds of recurrence. Therefore, we can immediately interpret Theorems A and $\mathrm{B}$ in terms of recurrence/transience as: $\left((0,1], F_{\lambda}, \Phi_{t}\right)$ is

- positive recurrent if $\lambda^{t} \in(0,1 / 2)$;

- null recurrent if $\lambda^{t}=1 / 2$;

- transient if $\lambda^{t} \in(1 / 2,1)$.

We can also compute the hyperbolic dimension

$$
\operatorname{dim}_{\text {hyp }}\left(F_{\lambda}\right):=\sup \left\{\operatorname{dim}_{H}(\Lambda): \Lambda \text { is compact, } F_{\lambda} \text {-invariant and } \Lambda \not \supset 0\right\}
$$

Our abuse of the word hyperbolic here is motivated by smooth one-dimensional dynamics, where 0 is the critical point. The hyperbolic dimension then refers to taking the supremum over all invariant closed sets that are bounded away from critical points, so at every iterate of the map, neighbourhoods of points in hyperbolic sets map to "large scale". In the usual cases of topologically transitive interval maps this value is 
equal to 1 , but the presence of dissipation in our systems can give $\operatorname{dim}_{\text {hyp }}\left(F_{\lambda}\right)<1$ for $\lambda \in(1 / 2,1)$, as in the next theorem. In addition, for $\lambda \in(0,1)$, we can define the escaping set as

$$
\Omega_{\lambda}:=\left\{x \in[0,1]: \lim _{n \rightarrow \infty} F_{\lambda}^{n}(x)=0\right\} .
$$

The result on the size of the escaping set stated below was proved in [SV]; our more general proof captures the hyperbolic dimension as well.

Theorem C. The Hausdorff dimension of hyperbolic and escaping sets are

$$
\operatorname{dim}_{h y p}\left(F_{1-\lambda}\right)=\operatorname{dim}_{H}\left(\Omega_{\lambda}\right)= \begin{cases}-\frac{\log 4}{\log [\lambda(1-\lambda)]} & \text { if } \lambda \leqslant \frac{1}{2} \\ 1 & \text { if } \lambda \geqslant \frac{1}{2}\end{cases}
$$

Our computations for Theorems $\mathrm{A}$ and $\mathrm{B}$ use an infinite matrix $A^{t}$ which models our system as an Markov chain. There is a corresponding infinite matrix $B^{t}$ which fits into the transfer operator approach. For $K \in \mathbb{N}$, we let $A_{K}^{t}$ and $B_{K}^{t}$ denote the corresponding truncated $K \times K$ matrices and for any matrix $D$ we let $\sigma(D)$ denote the spectral radius of $D$. In addition we will discuss topological pressure $P_{\text {top }}$ (based on $(n, \varepsilon)$-separated sets as introduced by Bowen, $\mathrm{Bo}$ and then used to define topological pressure in [R1] and [W1]) and Gurevich pressure $P_{G}$, which is particularly adapted to symbolic countable Markov chains. The next result brings together these various notions of pressure. It can be seen as a corollary of Theorems $\mathrm{A}$ and $\mathrm{B}$.

Corollary 1. For each $\lambda \in(0,1)$ and $t \in \mathbb{R}$,

$$
\begin{aligned}
P\left(\Phi_{t}\right) & =P_{G}\left(\Phi_{t}\right)=P_{\text {top }}\left(\Phi_{t}\right)=P_{\text {Conf }}\left(\Phi_{t}\right)=\log \sigma\left(B^{t}\right) \\
& =\lim _{K \rightarrow \infty} \log \sigma\left(B_{K}^{t}\right)=\lim _{K \rightarrow \infty} \log \sigma\left(A_{K}^{t}\right) .
\end{aligned}
$$

If $t=0$, then the above quantities are all equal to the topological entropy $\log 4$.

One can compare this result to [PRS, Proposition 1.2] for rational maps of the complex plane; specifically the equality between $P_{\mathrm{Conf}}$ and $P$.

The structure of this paper is as follows. First, in Section 2, we will prove that $F_{\lambda}$ has an acip, i.e., an $F_{\lambda}$-invariant probability measure absolutely continuous w.r.t. Lebesgue if and only if $\lambda \in\left(0, \frac{1}{2}\right)$. We take a probabilistic approach and introduce a random walk on a Markov chain perspective for these maps. Continuing the probabilistic approach, in Section 3 we introduce the more general $(t, p)$-conformal measure as a reference measure for $\left((0,1], F_{\lambda}\right)$, and investigate its thermodynamic properties including what we call conformal pressure. For the variational approach to pressure, we need the topological pressure on $F_{\lambda}$-invariant compact subsets of $(0,1]$, and to this end we use infinite matrices matrices $B^{t}$ and their $K \times K$ cropped versions $B_{K}^{t}$ and compute their leading eigenvalues in Section 4. This gives us also tools to compute the dimensions of hyperbolic and escaping sets (Theorem C) in Section 5. These various notions of pressure are discussed at length in Section 6, culminating in the proof of Theorem B. Finally, in Section 7, we show the null recurrence of $t$-conformal measure $m_{t}$ in the case that $\lambda^{t}=\frac{1}{2}$. 


\section{ACIPS FOR $F_{\lambda}$}

Now we will calculate the values of $\lambda$ for which there is an $F_{\lambda}$-invariant probability measure absolutely continuous w.r.t. Lebesgue (acip).

Theorem 1. The system $\left((0,1], F_{\lambda}\right)$ has an acip $\mu$ if and only if $\lambda \in\left(0, \frac{1}{2}\right)$ and in this case

$$
\mu\left(W_{i}\right)=\frac{1-2 \lambda}{\lambda}\left(\frac{\lambda}{1-\lambda}\right)^{i}
$$

If $\lambda \in\left(\frac{1}{2}, 1\right)$, Lebesgue measure is dissipative.

If $\lambda=\frac{1}{2}$, then Lebesgue measure is conservative, but there is no acip, so the system $\left((0,1], F_{\lambda}, \Phi_{1}\right)$ is null recurrent. We will return to this case in Section 7 , The proof of dissipativity is based on a random walk argument, similar to BKNS. Further work in this direction in non-linear setting can be found in [MS, SS], the latter inspired by questions in parabolic Kleinian groups.

Proof. For $F_{\lambda}$ (considered as a Markov process), let $\left(A_{i, j}\right)_{i, j}$ be the transition matrix corresponding to $F_{\lambda}$, and let $\left(v_{i}\right)_{i}$ be the invariant probability vector, i.e., left eigenvector with eigenvalue 1 . As $F_{\lambda}$ is a Markov map, and $F_{\lambda}$ is linear on each state $W_{k}$, we obtain $\mu\left(W_{k}\right)=v_{k}$. We have

$$
\left(A_{i, j}\right)_{i, j}=(1-\lambda)\left(\begin{array}{ccccccc}
1 & \lambda & \lambda^{2} & \lambda^{3} & \lambda^{4} & \ldots & \ldots \\
1 & \lambda & \lambda^{2} & \lambda^{3} & \lambda^{4} & \ldots & \ldots \\
0 & 1 & \lambda & \lambda^{2} & \lambda^{3} & \lambda^{4} & \ldots \\
0 & 0 & 1 & \lambda & \lambda^{2} & \lambda^{3} & \ldots \\
\vdots & \vdots & 0 & 1 & \lambda & \lambda^{2} & \ldots \\
\vdots & \vdots & \vdots & \vdots & \vdots & \vdots & \ddots
\end{array}\right)
$$

Suppose $v_{i}=\beta_{i} \rho^{i}$, where $\frac{1}{i} \log \beta_{i} \rightarrow 0$ as $i \rightarrow \infty$, so any exponential growth/decline of $v^{i}$ is captured in $\rho^{i}$. Then

$$
v_{N}=\sum_{i=1}^{N+1} v_{i} p_{i, N}=(1-\lambda)\left(\sum_{i=2}^{N+1} \rho^{i} \beta_{i} \lambda^{N+1-i}+\rho \beta_{1} \lambda^{N-1}\right) .
$$

Dividing by $\lambda^{N}$ we obtain

$$
\beta_{N}\left(\frac{\rho}{\lambda}\right)^{N}=(1-\lambda)\left(\lambda \sum_{i=2}^{N+1} \beta_{i}\left(\frac{\rho}{\lambda}\right)^{i}+\beta_{1} \frac{\rho}{\lambda}\right) .
$$

Write $\omega=\frac{\rho}{\lambda}$, then subtracting (5) for $N-1$ from (5) for $N$, and then dividing by $\omega^{N-1}$ gives

$$
(1-\lambda) \lambda \omega^{2} \beta_{N+1}-\omega \beta_{N}+\beta_{N-1}=0 .
$$

Solving the recurrence equation shows that $\beta_{N}=\beta_{1}\left(\alpha b_{+}^{N-1}+(1-\alpha) b_{-}^{N-1}\right)$ for $\alpha \in \mathbb{R}$ arbitrary, and

$$
b_{ \pm}=\frac{1 \pm \sqrt{1-4 \lambda(1-\lambda)}}{(1-\lambda) \lambda \omega}=\frac{1 \pm|1-2 \lambda|}{(1-\lambda) \lambda \omega}, \quad \text { so }\left\{\begin{array}{l}
b_{+}=\frac{1}{\lambda \omega}=\frac{1}{\rho} \\
b_{-}=\frac{1}{(1-\lambda) \omega}=\frac{\lambda}{(1-\lambda) \rho}
\end{array}\right.
$$


Therefore $\beta_{i}$ grows or decreases exponentially unless $\rho=1$ or $\rho=\frac{\lambda}{1-\lambda}$. The former gives $v_{i} \equiv \beta_{1}$, which does not give a probability vector and, moreover, is only a solution if $\lambda=\frac{1}{2}$. The only viable solution is therefore $\rho=\frac{\lambda}{1-\lambda}$, and direct inspection shows that taking $\alpha=1$ and $\beta_{i} \equiv \beta_{1}$ indeed solves (5). We normalise $\beta_{i} \equiv(1-\rho) / \rho=(1-2 \lambda) / \lambda$ to obtain the normalised solution

$$
v_{i}=\frac{1-2 \lambda}{\lambda}\left(\frac{\lambda}{1-\lambda}\right)^{i} \text { for } \lambda<\frac{1}{2} .
$$

Let us now show that Lebesgue measure is dissipative if $\lambda>\frac{1}{2}$. To this end, we consider the action of $F_{\lambda}$ as a random walk on the state space $\mathbb{N}$, and let $\chi_{n}(x)=j$ if $F_{\lambda}^{n}(x) \in \mathcal{W}_{j}$. The probability of going from state $i$ to $j$ is the $i, j$-th entry of $A$, and we are in particular interested in the conditional expectation (also called drift)

$$
\begin{aligned}
\operatorname{Dr}(\lambda) & :=\mathbb{E}\left(\chi_{n}-k \mid \chi_{n-1}=k\right)=-(1-\lambda)+\lambda(1-\lambda) \sum_{j \geqslant 1} j \lambda^{j} \\
& =-(1-\lambda)+\frac{\lambda^{2}}{(1-\lambda)}=\frac{2 \lambda-1}{1-\lambda} .
\end{aligned}
$$

Hence $\mathbb{E}\left(\chi_{n}-k \mid \chi_{n-1}=k\right)>0$ if $\lambda>\frac{1}{2}$. Define $Y_{i}=\left(\chi_{i}-\chi_{i-1}\right)-\mathbb{E}\left(\chi_{i}-\chi_{i-1}\right)$, then $\mathbb{E}\left(Y_{i}\right)=0$ and the second moment

$$
\sigma^{2}:=\mathbb{E}\left(Y_{n}^{2}\right)=(1-\lambda)+\lambda(1-\lambda) \sum_{i \geqslant 1} i^{2} \lambda^{i}
$$

is bounded and independent of $n$. Thus the Central Limit Theorem applies, so $\frac{1}{\sigma \sqrt{n}} \sum_{i=1}^{n} Y_{i}$ converges in distribution to a normally distributed random variable $\mathcal{Y}$. Also $\mathbb{E}\left(\chi_{i}-\right.$ $\left.\chi_{i-1}\right)=\sum_{k} \mathbb{E}\left(\chi_{i}-k \mid \chi_{i-1}=k\right) \mathbb{P}\left(\chi_{i-1}=k\right)=\operatorname{Dr}(\lambda)$. Therefore

$$
\chi_{n}=\chi_{0}+\sum_{i=1}^{n} Y_{i}+\sum_{i=1}^{n} \mathbb{E}\left(\chi_{i}-\chi_{i-1}\right) \geqslant \chi_{0}+\sigma \sqrt{n} \mathcal{Y}+n \operatorname{Dr}(\lambda) \rightarrow \infty \quad \text { a.s. }
$$

provided $\operatorname{Dr}(\lambda)>0$. This means that for $\lambda>\frac{1}{2}$, Lebesgue typical starting points will have $\chi_{n}(x) \rightarrow \infty$, and $F_{\lambda}^{n}(x) \rightarrow 0$ as $n \rightarrow \infty$.

\section{Conformal measures and conformal pressure for $F_{\lambda}$}

In this section we define and compute conformal pressure and combine it with the drift argument of the previous section to determine whether or not $(t, p)$-conformal measures are conservative. Throughout, maps and potentials are assumed to be Borel measurable.

\subsection{Definition of conformal measure.}

Definition 1. Given a dynamical system $(X, f)$ with potential $\phi: X \rightarrow \mathbb{R}$, a measure $m$ is called $\phi$-conformal if $m(f(A))=\int_{A} e^{-\phi} d m$ whenever $f: A \rightarrow f(A)$ is one-to-one on a measurable set $A$. 
Notice that if we perform a potential shift by a constant $p$ (i.e., $m(f(A))=\int_{A} e^{p-\phi} d m$ whenever $f: A \rightarrow f(A)$ is one-to-one), this will result in a $(\phi-p)$-conformal measure. Conformal measures corresponding to such shifted potentials are used to define $P_{\text {Conf }}(\phi)$ defined by (1). Since the canonical class of potentials for our system is $\left\{-t \log \left|F^{\prime}\right|: t \in\right.$ $\mathbb{R}\}$ (sometimes called the 'geometric potentials'), we will be interested in $\left(-t \log \left|F^{\prime}\right|-\right.$ $p$ )-conformal measures for some $p \in \mathbb{R}$. As mentioned in Section 1, for brevity we will call such a measure a $(t, p)$-conformal measure and denote it by $m_{t, p}$.

A measure $\mu$ on $X$ is called non-singular if $\mu(A)=0$ if and only if $\mu\left(f^{-1} A\right)=0$. A set $W \subset X$ is called wandering if the sets $\left\{f^{-n} W\right\}_{n=0}^{\infty}$ are disjoint.

Definition 2. Let $f: X \rightarrow X$ be a dynamical system. An $f$-non-singular measure $\mu$ is called conservative if every wandering set $W$ is such that $\mu(W)=0$.

A conservative measure satisfies the Poincaré Recurrence Theorem (see [Aa, p.17], or [S5, p.30]).

3.2. Computation of the conformal pressure for $F_{\lambda}$. The log of the function defined in the next lemma will turn out to be the conformal pressure.

Lemma 1. Given $\lambda \in[0,1)$, the map

$$
t \mapsto \psi(t):=\frac{(1-\lambda)^{t}}{1-\lambda^{t}}
$$

is analytic and strictly decreasing and strictly convex on $(0, \infty), \lim _{t \rightarrow 0} \psi(t)=\infty$, $\psi(1)=1, \psi^{\prime \prime}>0$ and $\lim _{t \rightarrow \infty} \psi(t)=0$.

Proof. Straight-forward calculus. Note that the derivatives are

$$
\psi^{\prime}(t)=\psi(t)\left[\log (1-\lambda)+\frac{\lambda^{t}}{1-\lambda^{t}} \log \lambda\right]<0
$$

and

$$
\psi^{\prime \prime}(t)=\psi(t)\left[\left(\log (1-\lambda)+\frac{\lambda^{t}}{1-\lambda^{t}} \log \lambda\right)^{2}+\frac{\lambda^{t}}{\left(1-\lambda^{t}\right)^{2}} \log ^{2} \lambda\right]>0
$$

for all $t \in(0, \infty)$.

Theorem 2. Fix $\lambda \in(0,1)$. Then for each $t>0$, the smallest $p \in \mathbb{R}$ such that there is $a(t, p)$-conformal measure $m_{t, p}$ is

$$
p=P_{\text {Conf }}\left(\Phi_{t}\right)= \begin{cases}\log \psi(t) & \text { if } \lambda^{t} \leqslant \frac{1}{2}, \\ \log 4\left[\lambda(1-\lambda]^{t}\right. & \text { if } \lambda^{t} \geqslant \frac{1}{2} .\end{cases}
$$

In this case, the conformal measure is given by

$$
m_{t, p}\left(W_{k}\right)= \begin{cases}\left(1-\lambda^{t}\right) \lambda^{t(k-1)} & \text { if } p=\log \psi(t) \text { and } \lambda^{t} \leqslant \frac{1}{2}, \\ {\left[(k-1)+\lambda^{-t}\left(1-\frac{k}{2}\right)\right]\left(\frac{1}{2}\right)^{k}} & \text { if } p=\log 4[\lambda(1-\lambda)]^{t} \text { and } \lambda^{t} \geqslant \frac{1}{2}\end{cases}
$$


Proof. Suppose that $m_{t, p}$ is a $(t, p)$-conformal measure. That is $L_{\Phi_{t}}^{*} m_{t, p}=e^{p} m_{t, p}$ for some $p \in \mathbb{R}$. Then

$$
\begin{aligned}
m_{t, p}\left(W_{k}\right) & =\int \mathbb{I}_{W_{k}} d m_{t, p}=\int L_{\Phi_{t}} e^{-p} \mathbb{I}_{W_{k}} d m_{t, p} \\
& =\int \sum_{F_{\lambda} y=x} e^{\Phi_{t}(y)-p} \mathbb{I}_{W_{k}}(y) d m_{t, p}(x) \\
& =\sum_{W_{k} \rightarrow W_{j}} e^{-p}\left|F_{\lambda}^{\prime}\right|_{W_{k}}^{-t} m_{t, p}\left(W_{j}\right),
\end{aligned}
$$

where $W_{k} \rightarrow W_{j}$ denotes the fact that $F_{\lambda}$ maps $W_{k}$ to $W_{j}$.

Therefore,

$$
m_{t, p}\left(W_{1}\right)=e^{-p}(1-\lambda)^{t} \sum_{j \geqslant 1} m_{t, p}\left(W_{j}\right) \quad \text { for } k=1
$$

and

$$
m_{t, p}\left(W_{k}\right)=e^{-p}[\lambda(1-\lambda)]^{t} \sum_{j \geqslant k-1} m_{t, p}\left(W_{j}\right) \quad \text { for } k \geqslant 2
$$

As we will see in the following claim, for some values of $(t, p)$, the conformal measure has a particularly simple form. As we prove below, these are the relevant measures when $\lambda^{t}<1 / 2$ and $p=P_{\text {Conf }}\left(\Phi_{t}\right)$.

Claim 1. If $\lambda^{t}<1$ then

$$
m_{t, p}\left(W_{k}\right)=\left(1-\lambda^{t}\right) \lambda^{t(k-1)}
$$

and $p=\log \psi(t)$ solve (8) and (91).

Proof. If $m_{t, p}\left(W_{j}\right)=C \gamma^{j}$, then

$$
C \gamma=m_{t, p}\left(W_{1}\right)=C e^{-p}(1-\lambda)^{t} \sum_{j \geqslant 1} \gamma^{j}=C e^{-p}(1-\lambda)^{t}\left(\frac{\gamma}{1-\gamma}\right),
$$

and hence $e^{-p}=\frac{1-\gamma}{(1-\lambda)^{t}}$. Similarly,

$$
C \gamma^{k}=m_{t, p}\left(W_{k}\right)=C e^{-p}[\lambda(1-\lambda)]^{t} \sum_{j \geqslant k-1} \gamma^{j}=C e^{-p}[\lambda(1-\lambda)]^{t}\left(\frac{\gamma^{k-1}}{1-\gamma}\right),
$$

and hence $e^{-p}=\frac{\gamma(1-\gamma)}{[\lambda(1-\lambda)]^{t}}$. Therefore, $\gamma=\lambda^{t}$ and $p=\log \psi(t)$. Finally, taking $C=\frac{1-\lambda^{t}}{\lambda^{t}}$ normalises $m_{t, p}$ to $\sum_{k} m_{t, p}\left(W_{k}\right)=1$. This proves Claim 1.

Next we will show that there is no $(t, p)$-conformal measure if $p<\log 4[\lambda(1-\lambda)]^{t}$. Suppose that there is a $(t, p)$-conformal measure $m_{t, p}$. Let $0<\varepsilon:=\log \left(4[\lambda(1-\lambda)]^{t}\right)-p$. We will see in the proof of Theorem 3 later on that the number of periodic points in $W_{1}$ of period $n$ exceeds $4^{(1-\varepsilon / 2) n}$ for $n$ sufficiently large. Let $X_{n}=W_{1} \cap F^{-n}\left(W_{1}\right)$ and let $\left\{Y_{n, i}\right\}_{i}$ be the collection of connected components of $X_{n}$. Since each $Y_{n, i}$ contains 
a periodic point of period $n$, there exists $C>0$ such that $\#\left\{Y_{n, i}\right\}_{i} \geqslant 4^{n(1-\varepsilon / 2)}$ for $n$ sufficiently large. To compute the measure of $Y_{n, i}$, we can use

$$
m_{t, p}\left(W_{1}\right)=\int_{Y_{n, i}} e^{p n}(1-\lambda)^{-t}[\lambda(1-\lambda)]^{-t(n-1)} d m_{t, p},
$$

so $m_{t, p}\left(Y_{n, i}\right)=C m_{t, p}\left(W_{1}\right) e^{-p n}[\lambda(1-\lambda)]^{t n}$ for some $C>0$. Using the cardinality of components $Y_{n, i}$, we find

$$
4^{n(1-\varepsilon / 2)} \cdot C e^{-p n}[\lambda(1-\lambda)]^{t n} m_{t, p}\left(W_{1}\right) \leqslant m_{t, p}\left(W_{1}\right),
$$

but the condition $p<\log 4\left[\lambda(1-\lambda]^{t}\right.$ makes this impossible to satisfy for large $n$.

From now on assume that $p \geqslant \log 4\left[\lambda(1-\lambda]^{t}\right.$. Subtracting (9) for $k+1$ from (9) for $k$ gives for $x_{k}:=m_{t, p}\left(W_{k}\right)$ :

$$
x_{k+1}-x_{k}+c x_{k-1}=0 \quad \text { where } c:=e^{-p}[\lambda(1-\lambda)]^{t} .
$$

Thus the roots of the generating equation $r^{2}-r+c=0$ are $r_{ \pm}=\frac{1 \pm \sqrt{1-4 c}}{2}$, and the general solution is $x_{k}=A_{+} r_{+}^{k}+A_{-} r_{-}^{k}$. We normalise so that the total mass is $\sum_{k} x_{k}=1$. Then (을 and (99) for $k=2$ give

$$
\left\{\begin{array}{l}
x_{1}=e^{-p}(1-\lambda)^{t}=c \lambda^{-t} \\
x_{2}=e^{-p} \lambda^{t}(1-\lambda)^{t}=c
\end{array}\right.
$$

Substituting $x_{k}=A_{+} r_{+}^{k}+A_{-} r_{-}^{k}$ in this equation and solving for $A_{ \pm}$gives

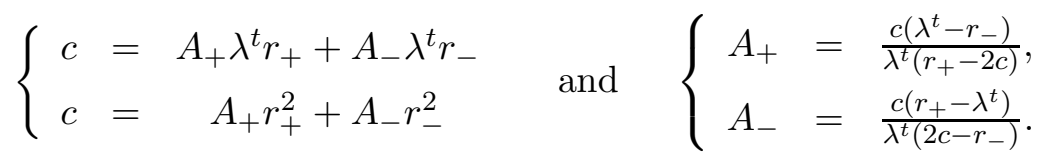

The form $r_{ \pm}=\frac{1 \pm \sqrt{1-4 c}}{2}$ and $c=e^{-p}[\lambda(1-\lambda)]^{t}$ implies the inequalities

$$
0<r_{-}<2 c=2 e^{-p} \lambda^{t}(1-\lambda)^{t} \leqslant \frac{1}{2}<r_{+}<1 \quad \text { and } \quad \lambda^{t}<r_{+},
$$

where the third inequality is strict if $p>\log 4[\lambda(1-\lambda)]^{t}$. This shows that $A_{-} \geqslant 0$.

If $\lambda^{t} \leqslant \frac{1}{2}$, then we find $A_{+} \geqslant 0$ when $\lambda^{t} \geqslant r_{-}$, which is precisely the case when $p \geqslant \psi(t)$. It is under this condition that $x_{k}>0$ for all $k \in \mathbb{N}$. If $\lambda^{t}=r_{-}(\operatorname{so} p=\log \psi(t))$, then $A_{+}=0$, and $A_{-}=\frac{1-\lambda^{t}}{\lambda^{t}}$. Hence the solution is the one given in Claim 1.

If $\lambda^{t}>\frac{1}{2}$, then clearly $\lambda^{t}>r_{-}$and hence $A_{+}>0$ for all allowed values of $p$, and $p=\log 4[\lambda(1-\lambda)]^{t}$ is smallest of these. In this case $r_{+}=r_{-}=\frac{1}{2}$, and this double root leads to a solution $x_{k}=A\left(\frac{1}{2}\right)^{k}+B k\left(\frac{1}{2}\right)^{k}$. Substitution in (11) gives $A=1-\frac{1}{\lambda^{t}}$ and $B=\frac{1}{2 \lambda^{t}}-1$.

Proof of Theorem $A$. The value of the smallest $p \in \mathbb{R}$ for which there is a $(t, p)$ conformal measure follows from Theorem 2. Now that we have established the existence of a $\left(t, P_{\text {Conf }}\left(\Phi_{t}\right)\right)$-conformal measure $m_{t}=m_{t, P_{\text {Conf }}\left(\Phi_{t}\right)}$, for $\lambda^{t}<1 / 2$ we can extend our transition probability matrix $A$ from (44) in Section 2 to a transition probability matrix 
with respect to $m_{t}$. Indeed, measured in $m_{t}$, the probability to move from state $W_{i}$ to $W_{j}$ is (using the definition of $(t, \log \psi(t))$-conformal measure)

$$
\begin{aligned}
\frac{m_{t}\left(W_{i} \cap F_{\lambda}^{-1}\left(W_{j}\right)\right)}{m_{t}\left(W_{i}\right)} & =\frac{\left.\left|F^{\prime}\right|_{W_{i}}\right|^{-t} \psi(t) m_{t}\left(W_{j}\right)}{\left.\left|F^{\prime}\right|_{W_{i}}\right|^{-t} \psi(t) \sum_{k \geqslant i-1} m_{t}\left(W_{k}\right)} \\
& =\frac{\left(1-\lambda^{t}\right) \lambda^{t(j-1)}}{\sum_{k \geqslant i-1}\left(1-\lambda^{t}\right) \lambda^{t(k-1)}} \\
& =\left(1-\lambda^{t}\right) \lambda^{t(j-i+1)}
\end{aligned}
$$

provided $j \geqslant i-1$. Therefore, if

$$
A^{t}=(1-\lambda)^{t}\left(\begin{array}{ccccccc}
1^{t} & \lambda^{t} & \lambda^{2 t} & \lambda^{3 t} & \ldots & \ldots & \ldots \\
1^{t} & \lambda^{t} & \lambda^{2 t} & \lambda^{3 t} & & & \\
0 & 1^{t} & \lambda^{t} & \lambda^{2 t} & \lambda^{3 t} & & \\
0 & 0 & 1^{t} & \lambda^{t} & \lambda^{2 t} & & \\
\vdots & & 0 & 1^{t} & \lambda^{t} & \lambda^{2 t} & \ldots \\
& & & & \ddots & \ddots & \ddots
\end{array}\right) .
$$

is the matrix $A$ in (4) with all entries raised to the power $t$, then $\psi^{-1}(t) A^{t}$ is a probability matrix and $\frac{m_{t}\left(W_{i} \cap F_{\lambda}^{-1}\left(W_{j}\right)\right)}{m_{t}\left(W_{i}\right)}=\psi^{-1}(t) A_{i, j}^{t}$. Now we are able to use the drift argument in the proof of Theorem 1 to conclude that the measure $m_{t}$ is conservative if $\lambda^{t}<1 / 2$.

Now to prove that any $(t, p)$-conformal measure is dissipative whenever $\lambda^{t}>1 / 2$ or $p>P_{\text {Conf }}\left(\Phi_{t}\right)$ (we leave the null recurrent case $\lambda^{t}=\frac{1}{2}$ and $p=\psi(t)$ to Section 7), we use the same drift argument for $m_{t, p}\left(W_{j}\right)=A_{+} r_{+}^{j}+A_{-} r_{-}^{j}$ as in the proof of Theorem 2 . (Note that $r_{ \pm}$and hence $A_{ \pm}$depends on $p$ via $c=e^{-p}[\lambda(1-\lambda)]^{t}$. Note also that $A_{+}>0$ for $p>P_{\text {Conf }}\left(\Phi_{t}\right)$.) Inserting this solution in (12), we find that the transition probability for $W_{i} \rightarrow W_{j}$ is

$$
\begin{aligned}
\frac{m_{t, p}\left(W_{i} \cap F_{\lambda}^{-1}\left(W_{j}\right)\right)}{m_{t, p}\left(W_{i}\right)} & =\frac{\left.\left|F^{\prime}\right|_{W_{i}}\right|^{-t} e^{p} m_{t, p}\left(W_{j}\right)}{\left.\left|F^{\prime}\right|_{W_{i}}\right|^{-t} e^{p} \sum_{k \geqslant i-1} m_{t, p}\left(W_{k}\right)} \\
& =\frac{A_{+} r_{+}^{j}+A_{-} r_{-}^{j}}{\sum_{k \geqslant i-1} A_{+} r_{+}^{j}+A_{-} r_{-}^{j}} \\
& =C_{i}\left(r_{+}^{j-i+1}+\alpha r_{-}^{j} r_{+}^{-i+1}\right)
\end{aligned}
$$

where $\alpha=\frac{A_{-}}{A_{+}}=\frac{\left(r_{+}-\lambda^{t}\right)\left(r_{+}-2 c\right)}{\left(\lambda^{t}-r_{+}\right)\left(2 c-r_{-}\right)}$, and $C_{i}=\left(1-r_{+}\right)\left(1+\alpha \frac{-r_{+}}{1-r_{-}}\left(\frac{r_{-}}{r_{+}}\right)^{i}\right)^{-1} \rightarrow 1-r_{+}$as $i \rightarrow \infty$. Since $r_{-}<\frac{1}{2}<r_{+}$, there is $i_{0}$ such that the drift

$$
C_{i} \sum_{j \geqslant i-1}(j-i-1)\left(r_{+}^{j-i+1}+\alpha r_{-}^{j} r_{+}^{-i+1}\right)=C_{i}\left(\frac{2 r_{+}-1}{\left(1-r_{+}\right)^{2}}+\alpha\left(\frac{r_{-}}{r_{+}}\right)^{i-1} \frac{2 r_{-}-1}{\left(1-r_{-}\right)^{2}}\right)
$$

is positive for all $i \geqslant i_{0}$. This means that whenever an orbit reaches a state $i \geqslant i_{0}$, the probability of wandering off to infinity afterwards is positive. Since $\chi_{n}(x) \geqslant i_{0}$ infinitely often $m_{t, p^{-}}$-a.e., it follows that $m_{t, p}$-typical orbits converge to 0 , proving that $m_{t, p}$ is dissipative. 
Next we discuss the $F_{\lambda}$-invariant measures that are absolutely continuous w.r.t. the $\left(t, P_{\text {Conf }}\left(\Phi_{t}\right)\right)$-conformal measure $m_{t}$

Proposition 1. If $\lambda^{t} \in(0,1 / 2)$ and $p=\psi(t)$, then there is an $F_{\lambda}$-invariant probability measure $\mu_{t} \ll m_{t}$ such that

$$
\mu_{t}\left(W_{j}\right)=v_{i}^{t}:=\frac{1-2 \lambda^{t}}{\lambda^{t}}\left(\frac{\lambda^{t}}{1-\lambda^{t}}\right)^{i}
$$

Proof. Direct inspection shows that the probability matrix $\psi^{-1}(t) A^{t}$ preserves the probability vector $\left(v_{j}^{t}\right)_{j \geqslant 1}$, provided $\lambda^{t} \in\left(0, \frac{1}{2}\right)$. For $\lambda^{t} \geqslant \frac{1}{2}$, the vector $\underline{v}^{t}$ is not summable, and the drift argument from Section 2 shows that $m_{t}$ is in fact dissipative for $\lambda^{t}>\frac{1}{2}$. (The case $\lambda^{t}=\frac{1}{2}$ is dealt with in Section [7)

Remark 1. Observe that in the case that there is an equilibrium state $\mu_{t}$ for $\Phi_{t}$, the density $\frac{d \mu_{t}}{d m_{t}}$ is unbounded. Moreover, $\mu_{t}$ is not a Gibbs state. These facts can be seen as follows.

Comparing Theorem 2 with formula (14), we obtain that $\frac{\mu_{t}\left(W_{n}\right)}{m_{t}\left(W_{n}\right)}=\frac{1-2 \lambda^{t}}{\left(1-\lambda^{t}\right)^{n+1}}$ and so, noticing that the density $\frac{d \mu_{t}}{d m_{t}}$ is constant on 1-cylinders, we have $\left.\frac{d \mu_{t}}{d m_{t}}\right|_{W_{n}} \rightarrow \infty$ as $n \rightarrow \infty$.

Now let

$$
\left[e_{0} \cdots e_{n-1}\right]=\left\{x \in(0,1]: F^{k} x \in W_{e_{j}} \text { for } 0 \leqslant j<n\right\} .
$$

be our notation for an n-cylinder set. To show that $\mu_{t}$ is not a Gibbs measure, we check that there is no distortion constant $C \geqslant 1$ such that for all cylinder sets $\left[e_{0} \cdots e_{n}\right]$

$$
\frac{1}{C} \leqslant \frac{\mu_{t}\left(\left[e_{0} \cdots e_{n}\right]\right)}{\exp \left(-n p+S_{n} \Phi_{t}(x)\right)} \leqslant C,
$$

where $\left.S_{n} \Phi_{t}(x)=\sum_{k=0}^{n-1} \Phi_{t}\left(F_{\lambda}^{k}\right)\right)$ is the $n$-th ergodic sum and $p=P_{\mathrm{Conf}}\left(\Phi_{t}\right)$.

Since $m_{t}$ is $(t, p)$-conformal,

$$
\begin{aligned}
m_{t}\left(\left[e_{0} \cdots e_{n}\right]\right) & =e^{-n p+S_{n} \Phi_{t}} m_{t}\left(F_{\lambda}^{n}\left(\left[e_{0} \cdots e_{n}\right]\right)\right) \\
& =e^{-n p+S_{n} \Phi_{t}} \frac{1-2 \lambda^{t}}{1-\lambda^{t}} \sum_{k \geqslant e_{n-1}-1}\left(\frac{1-\lambda^{t}}{\lambda^{t}}\right)^{k} \\
& =e^{-n p+S_{n} \Phi_{t}}\left(\frac{1-\lambda^{t}}{\lambda^{t}}\right)^{e_{n-1}-2} .
\end{aligned}
$$

Therefore $\frac{\mu_{t}\left(\left[e_{0} \cdots e_{n}\right]\right)}{e^{-n p+S_{n} \Phi_{t}}}=\frac{1-2 \lambda^{t}}{\left(1-\lambda^{t}\right)^{1+e_{0}}} \cdot\left(\frac{1-\lambda^{t}}{\lambda^{t}}\right)^{e_{n-1}-2}$ which is unbounded in $e_{n-1}$ and $e_{0}$.

\section{A SECOND APPROACH TO THERMODYNAMiC FORMALISM FOR $F_{\lambda}$}

In this section we employ the matrix $B^{t}$ in place of $A^{t}$ used previously. As we show in Section 6, this new matrix is more closely associated to the formalism of Sarig. 
There are two different ways of computing the sizes and their sums of $n$-cylinder sets, w.r.t. potential $-t \log \left|F_{\lambda}^{\prime}\right|$ (or equivalently, in terms of $t$-dimensional Hausdorff measure). One way is shown before Proposition 2, using the matrix $A^{t}$, and is based on first computing the sizes of 1-cylinders (namely for every $e_{0}=j$, there are two such cylinders, each of "t-dimensional" length $\left.\left|W_{j}\right|^{t}\right)$, and then considering which part of an $n$-cylinder belongs to a particular $(n+1)$-subcylinder. If $\underline{1}$ is the row-vector of ones and $\underline{w}_{t}$ is the row-vector with entries $\left|W_{j}\right|^{t}$, then this way gives

$$
\sum_{e_{0} \ldots e_{n-1}}\left|\left[e_{0} \ldots e_{n-1}\right]\right|^{t}=\underline{w}_{t} \cdot\left(A^{t}\right)^{n-1} \cdot \underline{1}^{T}
$$

where ${ }^{T}$ stands for the transpose of a vector or matrix.

The other way is by considering the slopes of $F_{\lambda}^{n}$ and the " $t$-dimensional" length of the $F_{\lambda}^{n}$-image of each cylinder. The matrix $B^{t}$ contains the inverse slopes (raised to the power $t$ )

$$
B^{t}=(1-\lambda)^{t}\left(\begin{array}{ccccccc}
1 & 1 & 1 & 1 & 1 & \ldots & \ldots \\
\lambda^{t} & \lambda^{t} & \lambda^{t} & \lambda^{t} & \lambda^{t} & \ldots & \ldots \\
0 & \lambda^{t} & \lambda^{t} & \lambda^{t} & \lambda^{t} & \lambda^{t} & \ldots \\
0 & 0 & \lambda^{t} & \lambda^{t} & \lambda^{t} & \lambda^{t} & \ldots \\
\vdots & \vdots & 0 & \lambda^{t} & \lambda^{t} & \lambda^{t} & \ldots \\
\vdots & \vdots & \vdots & \vdots & \vdots & \vdots & \ddots
\end{array}\right) .
$$

This way gives

$$
\sum_{e_{0} \ldots e_{n-1}}\left|\left[e_{0} \ldots e_{n-1}\right]\right|^{t}=\underline{1} \cdot\left(B^{t}\right)^{n-1} \cdot \underline{w}_{t}^{T} .
$$

For this to make sense, it is important for both matrices to be related, and in fact, if $A_{K}^{t}$ and $B_{K}^{t}$ are the $K \times K$-left-upper matrices of $A^{t}$ and $B^{t}$ respectively, then we have the following result.

Lemma 2. For each $K$, the characteristic polynomials of $A_{K}^{t}$ and $B_{K}^{t}$ coincide.

Proof. The matrix $A_{K}^{t}$ can be transformed into the matrix $B_{K}^{t}$ by a sequence of $2(K-$ 1 ) elementary column and row operations. Namely, we multiply the $i$-th column by $\lambda^{-(i-1) t}$ and the $i$-th row by $\lambda^{(i-1) t}$. This has no effect on diagonal elements. Hence, the same operations transform $\left(A_{K}^{t}-x I\right)$ into $\left(B_{K}^{t}-x I\right)$, and the effects on the determinant cancel out.

This means that we can dispense with the distinction between $A^{t}$ and $B^{t}$ in characteristic polynomials

$$
\alpha_{t, K}(s)=(1-\lambda)^{-t} \operatorname{det}\left(A_{K}^{t}-s I\right)=(1-\lambda)^{-t} \operatorname{det}\left(B_{K}^{t}-s I\right) .
$$

In fact, $\alpha_{t, K}$ satisfies the recursive formula $\alpha_{t, K}(s)=-s \alpha_{t, K-1}(s)-s \lambda^{t} \alpha_{t, K-2}(s)(s)$ which leads to

$$
\alpha_{t, K}(s)=\sum_{j=0}^{\lfloor(K+1) / 2\rfloor}\left(\begin{array}{c}
K+1-j \\
j
\end{array}\right)(-1)^{K+1-j} s^{n-j} \lambda^{t j},
$$



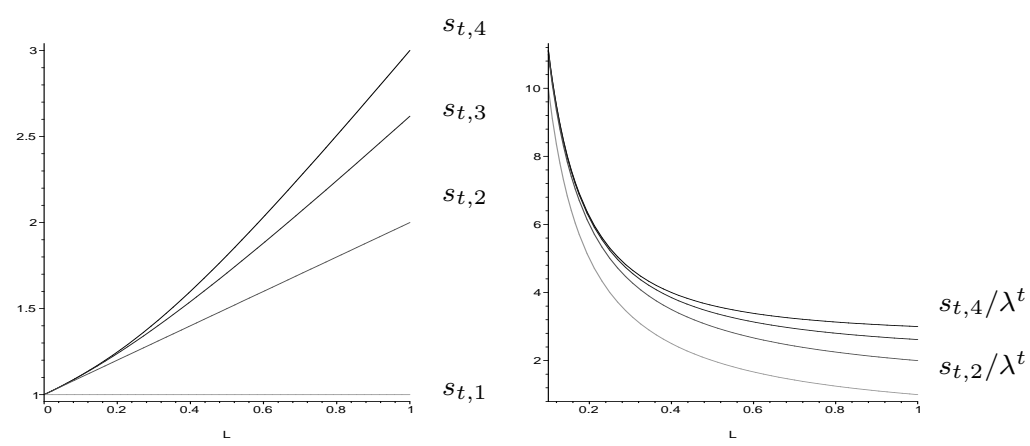

Figure 1. Graphs of the eigenvalues $s_{t, j}$ (left) of $A_{j}^{t}$ and $s_{t, j} / \lambda^{t}$ (right) for $j=1, \ldots, 4$ as function of $\lambda^{t} \in[0,1]$.

that is, the coefficients of $\alpha_{0, K}$ are signed elements of Pascal's triangle along the $K$-th north-east-east diagonal. Letting $s_{t, K}$ be the corresponding leading eigenvalue, we have

$$
\begin{aligned}
\alpha_{t, 1}(s) & =-s+1 & s_{t, 1} & =1 \\
\alpha_{t, 2}(s) & =s^{2}-\left(\lambda^{t}+1\right) s & s_{t, 2} & =\lambda^{t}+1 \\
\alpha_{t, 3}(s) & =-s^{3}+\left(2 \lambda^{t}+1\right) s^{2}-\lambda^{t} s & s_{t, 3} & =\frac{2 \lambda^{t}+1+\sqrt{4 \lambda^{2 t}+1}}{2} \\
\alpha_{t, 4}(s) & =s^{4}-\left(3 \lambda^{t}+1\right) s^{3}+\left(\lambda^{2 t}+2 \lambda^{t}\right) s^{2} & s_{t, 4} & =\frac{3 \lambda^{t}+1+\sqrt{5 \lambda^{2 t}-2 \lambda^{t}+1}}{2}
\end{aligned}
$$

Remark 2. For each $K$, the non-negative matrices $A_{K+1}^{t}$ and $B_{K+1}^{t}$ are at least as large element-wise as the matrices $A_{K}$ and $B_{K}$ augmented with an extra row and column of zeroes. From this it follows that the leading eigenvalues are increasing in $K$ for all $t$.

One can verify by induction that $r^{-K} \alpha_{t, K}(r)=(-1)^{K} \lambda^{K t}$ for $r=1 /\left(1-\lambda^{t}\right)$, which suggests that the leading root of $\alpha_{t, K}$ is $x_{t, K}(t)=r(1-\lambda)^{t}<\psi(t)$, and potentially in the limit $x(t)=\lim _{K \rightarrow \infty} x_{t, K}=\psi(t)$. The next proposition confirms this for $\lambda^{t} \leqslant \frac{1}{2}$.

Theorem 3. The limit of the leading eigenvalues of the matrices $B_{K}^{t}$ is

$$
x(t):=\lim _{K \rightarrow \infty} x_{t, K}= \begin{cases}\psi(t)=\frac{(1-\lambda)^{t}}{1-\lambda^{t}} & \text { if } \lambda^{t} \leqslant \frac{1}{2} \quad \text { i.e., } t \geqslant t_{0}:=\frac{-\log 2}{\log \lambda} ; \\ 4 \lambda^{t}(1-\lambda)^{t} & \text { if } \lambda^{t} \geqslant \frac{1}{2} \quad \text { i.e., } t \leqslant t_{0} .\end{cases}
$$

Hence $\log x(t)$ is analytic, except at $t=t_{0}$ where it is $C^{1}$ but not $C^{2}$. Furthermore,

$$
\log x\left(t_{1}\right)=0 \quad \text { for } \quad t_{1}= \begin{cases}1 & \text { if } \lambda \leqslant \frac{1}{2} \\ -\frac{\log 4}{\log [\lambda(1-\lambda)]} & \text { if } \lambda \geqslant \frac{1}{2}\end{cases}
$$

Proof. For fixed $K \in \mathbb{N}$, write $x_{t, K}=s_{t, K}(1-\lambda)^{t}=r_{t, K} \lambda^{t}(1-\lambda)^{t}$. For brevity, we will write $r=r_{t, K}$ and $r_{\infty}=\lim _{K \rightarrow \infty} r_{t, K}$. 

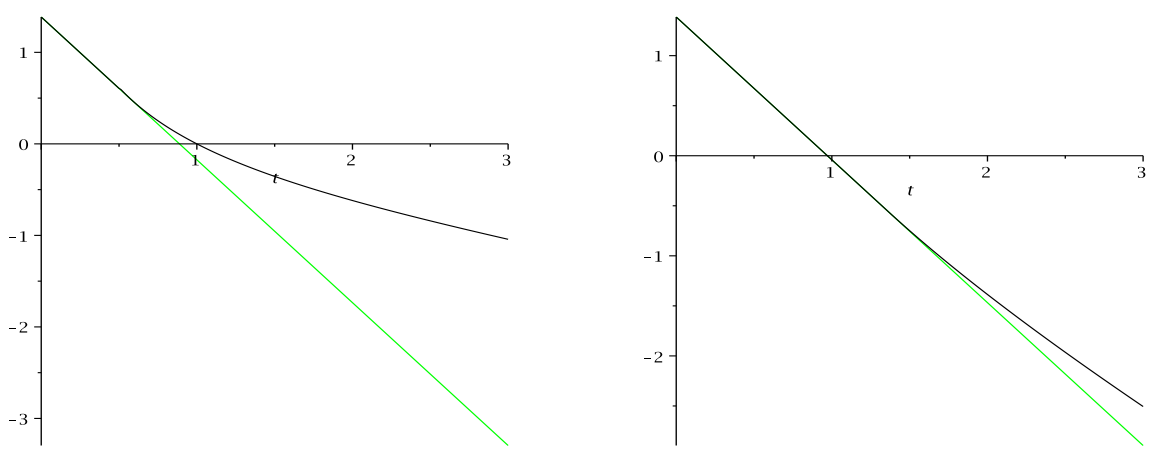

Figure 2. Graphs of $P\left(-t \log \left|F_{\lambda}^{\prime}\right|\right)$ for $\lambda=0.3$ with $t_{0} \approx 0.5, t_{1}=1$ (left) and $\lambda=0.6$ with $t_{0} \approx 1.4, t_{1} \approx 0.94$ (right). In either case, $t \mapsto$ $t \log (1-\lambda)$ is the oblique asymptote on the right. Since $\frac{1}{\lambda(1-\lambda)} \geqslant\left|F^{\prime}\right| \geqslant$ $\frac{1}{1-\lambda}$, we always have $\log 4+t \log (1-\lambda) \geqslant P\left(\Phi_{t}\right) \geqslant \log 4+t \log [\lambda(1-\lambda)]$ (drawn in light colour).

Let $\underline{v}=\left(v_{1}, v_{2}, \ldots\right)$ be the left eigenvector of $(1-\lambda)^{-t} B_{K}^{t}$ for eigenvalue $s_{t, K}$, scaled such that $v_{1}=1$. By the Perron-Frobenius Theorem, we know that $v_{j}>0$ for all $j$. The shape of the columns of $B_{K}^{t}$ imply that $v_{j} \leqslant v_{j+1}$ for all $j \leqslant K$, and in fact $v_{K-1}=v_{K}$ (as the last two columns of $B_{K}^{t}$ are identical). We can recursively solve $v_{2}=r-\lambda^{-t}$ and $v_{3}=(r-1) v_{2}-\lambda^{-t}=r^{2}-\left(1+\lambda^{-t}\right) r$, and since $v_{2}>0$, this already gives $s_{t, K}=\lambda^{t} r>\lambda^{t}\left(1+\lambda^{-t}\right)=\left(1+\lambda^{t}\right)$. Similarly, $v_{3} \geqslant v_{2}$ implies that $r^{2}-\left(2+\lambda^{-t}\right) r+\lambda^{-t} \geqslant 0$, whence $r \geqslant \frac{1}{2}\left(2+\lambda^{-t}+\sqrt{4+\lambda^{-2 t}}\right)$. The general term is

$$
v_{j}=(r-1) v_{j-1}-\sum_{k=2}^{j-2} v_{k}-\lambda^{-t} \quad \text { for } 3 \leqslant j \leqslant K .
$$

If we write $v_{j}=a_{j}-b_{j} \lambda^{-t}$, we find

$$
\begin{cases}a_{n}=r a_{n-1}-\sum_{k=1}^{n-1} a_{k}+1 & a_{2}=r, a_{1}=1, a_{0}=0 \\ b_{n}=r b_{n-1}-\sum_{k=1}^{n-1} b_{k}+1 & b_{2}=1, b_{1}=0 .\end{cases}
$$

An induction proof then gives that

$$
a_{n}=r\left(a_{n-1}-a_{n-2}\right) \quad b_{n}=a_{n-1} .
$$

This recursive formula has the characteristic equation $\mu^{2}-\mu r-r=0$, with solutions $\mu_{ \pm}=\frac{1}{2}\left(r \pm \sqrt{r^{2}-4 r}\right)$. Writing $a_{n}=A_{+} \mu_{+}^{n}+A_{-} \mu_{-}^{n}$, we readily find that $A_{+}=-A_{-}=$ $1 / \sqrt{r^{2}-4 r}$, and therefore

$$
a_{n}= \begin{cases}\frac{1}{\sqrt{r^{2}-4 r}}\left(\mu_{+}^{n}-\mu_{-}^{n}\right) & \text { if } r>4 ; \\ n 2^{n-1} & \text { if } r=4 ; \\ \frac{2 \sin (\alpha n)}{\sqrt{4 r-r^{2}}} r^{n / 2} & \text { if } r<4 \text { and } \tan \alpha=\sqrt{\frac{4}{r}-1} .\end{cases}
$$


Remark 3. Putting in the numbers for $a_{1}=1, a_{2}=r, a_{n}=r\left(a_{N-1}-a_{n-2}\right)$, we get

$$
\begin{aligned}
& a_{3}=r^{2}-r, \\
& a_{4}=r^{3}-2 r^{2}, \\
& a_{5}=r^{4}-3 r^{3}+r^{2}, \\
& a_{6}=r^{5}-4 r^{4}+3 r^{3}, \\
& a_{7}=r^{6}-5 r^{5}+6 r^{4}-r^{3}, \\
& a_{8}=r^{7}-6 r^{6}+10 r^{5}-4 r^{4},
\end{aligned}
$$

which are the signed entries along the north-east-east diagonals in Pascal's triangle, so

$$
a_{K}(x)=\sum_{j=0}^{\lfloor(K-1) / 2\rfloor}\left(\begin{array}{c}
K+1-j \\
j
\end{array}\right)(-1)^{i} r^{K-j} .
$$

I: First assume that $\lambda^{t}<\frac{1}{2}$, so $\lambda^{-t}>2$.

If $r_{\infty}>4$, then $a_{j}$ will grow exponentially fast with rate $\mu_{+} \geqslant r / 2 \geqslant 2$, and

$$
v_{j}=a_{j}-\lambda^{-t} b_{j}=\frac{1}{\sqrt{r^{2}-4 r}}\left[\left(\mu_{+}-\lambda^{-t}\right) \mu_{+}^{j-1}+\left(\lambda^{-t}-\mu_{-}\right) \mu_{-}^{j-1}\right] .
$$

If $\mu_{+}>\lambda^{-t}$, then indeed $v_{j}>0$ for all $j$, but we get a contradiction via the following argument (which we will call argument $A$ ): Since $v$ is a left eigenvector for eigenvalue $r_{t, K}=r \lambda^{t}$

$$
r v_{K-2}=\sum_{j=2}^{K-1} v_{j}+v_{1} \lambda^{-t} \quad \text { and } \quad r v_{K-1}=\sum_{j=2}^{K} v_{j}+v_{1} \lambda^{-t} .
$$

Subtract the two equations, and recall that $v_{K}=v_{K-1}$. Then $r\left(v_{K-1}-v_{K-2}\right)=v_{K}=$ $v_{K-1}$, so $r \sim \mu_{+} /\left(\mu_{+}-1\right) \leqslant 2$, contrary to the assumption that $r \geqslant 4$.

The case $\mu_{+}=\lambda^{-t}$ implies that

$$
r_{\infty}=\frac{\lambda^{-t}}{1-\lambda^{t}} \quad\left(\text { whence } x=(1-\lambda)^{t} \lambda^{t} r_{\infty}=\psi(t)\right) \quad \text { and } \quad \mu_{-}=\frac{1}{1-\lambda^{t}}>1 .
$$

Note that indeed $r_{\infty} \geqslant 4$ if $\lambda^{t} \leqslant \frac{1}{2}$. In this case,

$$
v_{j}=\frac{\lambda^{-t}-\mu_{-}}{\sqrt{r^{2}-4 r}} \mu_{-}^{j-1}=\frac{1}{1-\lambda^{t}} \mu_{-}^{j-1}
$$

and argument $\mathrm{A}$ gives $r_{\infty}=\mu_{-} /\left(\mu_{-}-1\right)=\lambda^{-t}$, which still looks like a contradiction. However, argument A relies on having a finite matrix $B_{K}^{t}$ with the two last columns identical. If $K<\infty$, then we can in fact still take $\mu_{+}<\lambda^{-t}$ close to $\lambda^{-t}$, because (20) only implies that $v_{j}<0$ for large $j$. This gives $x_{t, K}<\psi(t)$, but $\mu_{+} \rightarrow \lambda^{-t}$ and $x_{t, K} \rightarrow \psi(t)$ as $K \rightarrow \infty$.

If $r_{\infty}=4$, then $v_{j}=j 2^{j}-\lambda^{-t}(j-1) 2^{j-1}=2^{j}\left[1-\frac{\lambda^{-t}-2}{2}(j-1)\right]$, and this is negative as soon as $j>1 /\left(1-2 \lambda^{t}\right)$. 
If $r_{\infty}<4$, then

$$
v_{j}=\frac{2}{\sqrt{4 r-r^{2}}} r^{(n-1) / 2}\left[r^{1 / 2} \sin (\alpha j)-\lambda^{-t} \sin (\alpha(j-1))\right],
$$

and this becomes negative when $\sin (\alpha j)<\sin (\alpha(j-1))$.

So in the limit $K \rightarrow \infty$, this rules out $r \leqslant 4$, and therefore $x_{t}=\lim _{K \rightarrow \infty} x_{t, K}=\psi(t)$ as claimed.

II: Assume $\lambda^{t}=\frac{1}{2}$, so $\lambda^{-t}=2$.

Now $r_{\infty}>4$ gives that $\mu_{+}>2$, so $v_{j}=\mu_{+}^{j-1}\left(\mu_{+}-\lambda^{-t}\right)+\mu_{-}^{j-1}\left(\lambda^{-t}-\mu_{-}\right)>0$; in fact $v_{j}$ increases exponentially fast and $v_{j} / v_{j-1} \rightarrow \mu_{+}$. Therefore argument $\mathrm{A}$ implies that for large $K, r \sim \mu_{+} /\left(\mu_{+}-1\right) \leqslant r /(r-2) \leqslant r / 2$, which is a contradiction.

The case $r_{\infty}<4$ leads to a contradiction in the same way as in Case I. So the remaining possibility is $r_{\infty}=4$. In this case $v_{j}=2^{j}$, and argument $\mathrm{A}$ would again give a contradiction, if we could apply it to an infinite matrix. For finite matrices $B_{K}^{t}$, taking $r<4$ gives $v_{j}=\frac{2}{\sqrt{4 r-r^{2}}} r^{(n-1) / 2}\left[r^{1 / 2} \sin (\alpha j)-2 \sin (\alpha(j-1))\right]$ as in (21), but if $r^{1 / 2}$ is sufficiently close to 2 and $\alpha=\sqrt{\frac{4}{r}-1}$ sufficiently close to 0 , then $v_{j}$ is still positive for $j \leqslant K$. Hence $r \rightarrow 4$ and $x_{t, K} \rightarrow 1$ as $K \rightarrow \infty$,

III: The final case is $\lambda^{t}>\frac{1}{2}$, so $\lambda^{-t}<2$.

Now $r_{\infty}>4$ fails by argument $\mathrm{A}$ as in Case I, and if $r_{\infty}<4$, then $\alpha=\sqrt{\frac{4}{r}-1}$ is bounded away from 0 uniformly in $K$. Since $v_{j}$ is as given by (21), it becomes negative for $j$ sufficiently large (and independently of $K$ ), namely when $\sin (\alpha j) \leqslant 0 \leqslant$ $\sin (\alpha(j-1))$,

Therefore $r_{\infty}=4$, as it is the only way allowing $\alpha \rightarrow 0$ as $K \rightarrow \infty$.

This completes the proof (17). Direct computation shows that at $t=t_{0}$, the left and right derivatives are $\log [\lambda(1-\lambda)]$, but the second left derivative is 0 and right second derivative is $2 \log ^{2} \lambda$. Therefore $t \mapsto \log x(t)$ is $C^{1}$ but not $C^{2}$. The formula for $t_{1}$ is straightforward.

\section{The SIZE OF HYPERBOLIC AND ESCAPING SETS}

In this section we compute the Hausdorff dimension of hyperbolic sets (Proposition 2) and the escaping set (Proposition 3 ), which combined prove Theorem C.

Proposition 2. Let $\Lambda_{K}=\left\{x \in(0,1]: F^{i}(x) \notin \cup_{k>K}\left(W_{k}\right)\right.$ for all $\left.i \geqslant 0\right\}$. Then

$$
\lim _{K \rightarrow \infty} \operatorname{dim}_{H}\left(\Lambda_{K}\right)= \begin{cases}1 & \text { if } \lambda \leqslant \frac{1}{2} \\ -\frac{\log 4}{\log [\lambda(1-\lambda)]} & \text { if } \lambda \geqslant \frac{1}{2} .\end{cases}
$$

Proof. The cylinder sets $\left\{\left[e_{0} \ldots e_{n-1}\right]\right\}_{e_{i} \leqslant K}$ form a cover of $\Lambda_{K}$ of diameter tending to 0 as $n \rightarrow \infty$. Since equations (15) and (16) hold for the $K \times K$ matrices $A_{K}^{t}$ and $B_{K}^{t}$ 
as well, we find

$$
H_{t, K}^{n}:=\sum_{e_{i} \leqslant K}\left|\left[e_{0} \ldots e_{n-1}\right]\right|^{t}=\underline{w}_{t} \cdot\left(A_{K}^{t}\right)^{n-1} \cdot \underline{1}^{T}=\underline{1} \cdot\left(B_{K}^{t}\right)^{n-1} \cdot \underline{w}_{t}^{T} .
$$

These quantities develop exponentially in $n$ according to the leading eigenvalue $x_{t, K}$ of $A_{K}^{t}\left(\right.$ or $\left.B_{K}^{t}\right)$. Therefore $\inf \left\{t: H_{t, K}^{n}<\infty\right.$ for all $\left.n \in \mathbb{N}\right\}$ coincides with the first zero of $t \mapsto \log x_{t, K}$. Taking the limit $K \rightarrow \infty$, we find by Theorem 3 that

$$
\lim _{K \rightarrow \infty} \operatorname{dim}_{H}\left(\Lambda_{K}\right) \leqslant t_{1}= \begin{cases}1 & \text { if } \lambda \leqslant \frac{1}{2} \\ -\frac{\log 4}{\log [\lambda(1-\lambda)]} & \text { if } \lambda \geqslant \frac{1}{2}\end{cases}
$$

Now for a lower bound, we first treat the case $\lambda \geq \frac{1}{2}$. Take $\hat{\Lambda}_{K+1}=\left\{x \in \Lambda_{K+1}\right.$ : $F_{\lambda}(x) \notin W_{1}$ for all $\left.n \geqslant 0\right\}$. Observe that $\left\{\left[e_{0} \ldots e_{n-1}\right]\right\}_{2 \leqslant e_{i} \leqslant K+1}$ is a cover of $\hat{\Lambda}_{K+1}$ of the same cardinality as $\left\{\left[e_{0} \ldots e_{n-1}\right]\right\}_{1 \leqslant e_{i} \leqslant K}$, and consisting of intervals of length $[\lambda(1-\lambda)]^{n-1}\left|W_{e_{n-1}}\right|$. Moreover, $\hat{\Lambda}_{K+1}$ is a self-similar Cantor set (with bounded ratios between bridges and gaps) and by fairly standard arguments one can conclude that its dimension is given by the zero of the leading eigenvalue $t \mapsto \log \hat{x}_{t, K+1}$ of $\hat{A}_{K+1}^{t}$, which is defined as $A_{K+1}^{t}$ with the first row and column removed. The same argument as used in Lemma 2 shows that $\hat{A}_{K+1}^{t}$ and $\hat{B}_{K+1}^{t}$ (which is defined as $B_{K+1}^{t}$ with the first row and column removed) have the same characteristic polynomial, but

$$
\hat{B}_{K+1}^{t}=\lambda^{t}(1-\lambda)^{t}\left(\begin{array}{ccccccc}
1 & 1 & 1 & 1 & 1 & \ldots & \ldots \\
1 & 1 & 1 & 1 & 1 & \ldots & \ldots \\
0 & 1 & 1 & 1 & 1 & 1 & \ldots \\
0 & 0 & 1 & 1 & 1 & 1 & \ldots \\
\vdots & \vdots & 0 & 1 & 1 & 1 & \ldots \\
\vdots & \vdots & \vdots & \vdots & \vdots & \vdots & \ddots
\end{array}\right)=\lambda^{t}(1-\lambda)^{t} B_{K}^{0}
$$

as in Theorem 3. Therefore the leading eigenvalue $\hat{x}_{t, K}=\lambda^{t}(1-\lambda)^{t} x_{0, K} \rightarrow 4 \lambda^{t}(1-\lambda)^{t}$ as $K \rightarrow \infty$. The required lower bound for the zero $t_{1}$ follows, proving (22).

Now for the case $\lambda<\frac{1}{2}$, write $F=F_{\lambda}$ (we will use $\lambda$ for leading eigenvalue shortly). Then as in Theorem 4, $P(-\log |D F|)=0$. Moreover, $P(-t \log |D F|)>0$ for any $t<1$. Truncate the system at symbol $K$ to get pressure $P_{K}$ and note that by Sarig's theory [S1] for $K$ large enough $P_{K}(-t \log |D F|)>0$. There must be an equilibrium state $\mu$ on this truncated system for this potential and it has

$$
\operatorname{dim}_{H} \mu=\frac{h(\mu)}{\lambda(\mu)}=\frac{P_{K}(-t \log |D F|)}{\lambda(\mu)}+t>t
$$

which implies $\operatorname{dim}_{H}\left(\Lambda_{K}\right)>t$ also. Recalling that $t$ was an arbitrary number $<1$, we obtain the lower bound 1 .

The following proposition, giving a different proof to the result of Stratmann \& Vogt [SV], proves the other part of Theorem C.

Proposition 3. The dimension $\operatorname{dim}_{H}\left(\Omega_{\lambda}\right)$ is given by

$$
\operatorname{dim}\left(\Omega_{\lambda}\right)= \begin{cases}-\log 4 / \log [\lambda(1-\lambda)] & \text { if } \lambda \leqslant \frac{1}{2} \\ 1 & \text { if } \lambda \geqslant \frac{1}{2}\end{cases}
$$


Proof. Let $\Omega_{\lambda}^{\prime}=\left\{x \in \Omega_{\lambda}: F_{\lambda}^{n}(x) \notin W_{1}\right.$ for all $\left.n \geqslant 0\right\}$. Since $\Omega_{\lambda}=\cup_{n} F_{\lambda}^{-n}\left(\Omega_{\lambda}^{\prime}\right)$, it suffices to compute the Hausdorff dimension of $\Omega_{\lambda}^{\prime}$. We can use (16) to approximate the $t$-dimensional Hausdorff mass of $\Omega_{\lambda}^{\prime}$, and if we replace the first row of $A^{t}=A_{\lambda}^{t}$ by zeroes, this has no effect on the estimate, because by definition no point in $\Omega_{\lambda}^{\prime}$ ever "uses" the first state.

For $\lambda>\frac{1}{2}$ we know that $F_{\lambda}$ is dissipative, so $\lim _{n \rightarrow \infty} F_{\lambda}^{n}(x)=0$ Lebesgue-a.e. $x$, and the Hausdorff dimension of such points is certainty 1, proving the proposition in this case. Therefore take $\lambda<\frac{1}{2}$. Let $\eta \in\left(0, \frac{\log 2}{\log \lambda} \log \frac{\lambda}{1-\lambda}\right)$ be small and $\gamma \in\left(\frac{1}{2}, 1-\lambda\right)$. Since $\gamma$ corresponds to dissipative behaviour, the Lebesgue measure of $\Omega_{\gamma}^{\prime}$ is positive, so its Hausdorff dimension is 1 . Recall from (7) that the drift $\operatorname{Dr}(\gamma)=\frac{2 \gamma-1}{1-\gamma}$, which tends to 0 as $\gamma \rightarrow \frac{1}{2}$. Recall that $\chi_{n}(x)=k$ if $F_{\lambda}^{n}(x) \in W_{k}$. Measured w.r.t. Lebesgue measure, the vast majority of points satisfy $\chi_{n}(x) \sim n \operatorname{Dr}(\gamma)$, so that in fact the set of points $\Omega_{\gamma}^{\prime \prime}=\left\{x \in \Omega_{\gamma}^{\prime}: \chi_{n}(x) \leqslant 10 \operatorname{Dr}(\gamma) n\right.$ for all $n$ sufficiently large $\}$ has positive Lebesgue measure.

Note that the sets $\Omega, \Omega^{\prime}$ and $\Omega^{\prime \prime}$ depend on the parameter $\lambda$ (or $\gamma$ ), but the codes, or equivalently the sequences $\chi_{n}(x)$ for $x$ in these sets are independent of the parameter.

Since $\operatorname{dim}_{H}\left(\Omega_{\gamma}^{\prime \prime}\right)=1$, for each $u<1$, we can find a mesh $\varepsilon$ covers $\mathcal{U}_{\varepsilon}$ of $\Omega_{\gamma}$ using cylinder sets of variable lengths, such that $\sum_{C \in \mathcal{U}_{\varepsilon}}|C|^{u}$ diverges as $\varepsilon \rightarrow 0$.

The idea for $\lambda<\frac{1}{2}$ is now to use a cover of cylinders that for $\gamma$ represents positive measure, and hence finite $u$-dimensional Hausdorff mass for all $u<1$. Choose $t=$ $\frac{u \log [\gamma(1-\gamma)]+\eta \log \lambda}{\log [\lambda(1-\lambda]} \in(0,1)$, so

$$
[\lambda(1-\lambda)]^{t}=[\gamma(1-\gamma)]^{u} e^{u \eta}
$$

Because $\eta<\frac{\log 2}{\log \lambda} \log \frac{\lambda}{1-\lambda}$ we obtain for and $\gamma$ sufficiently close to $\frac{1}{2}$ that

$$
\log \frac{\lambda^{t}}{\gamma^{u}}=t \log \lambda-u \log \gamma=u \frac{\log (1-\gamma) \log \lambda-\log \gamma \log (1-\lambda)+\eta}{\log [\lambda(1-\lambda)]}<0,
$$

so $\lambda^{t}<\gamma^{u}$. We use the same covers $\mathcal{U}_{\varepsilon}$ and note that $t$-conformal length of cylinders $C$ for parameter $\lambda$ coincides with the $u$-conformal length of $C$ for parameter $\gamma$. Hence, indicating the parameter used in computing the length of intervals by a subscript, we get by (10) that for an $n$-cylinder $C$ such that $F_{\lambda}^{n-1}(C)=W_{\chi_{n}(C)}$ :

$$
|C|_{\lambda}^{t}=[\lambda(1-\lambda)]^{t(n-1)} m_{t}\left(W_{\chi_{n}(C)}\right)=[\lambda(1-\lambda)]^{t n} \lambda^{t\left(\chi_{n}(C)-2\right)}
$$

and similar for $|C|_{\gamma}^{u}$. Summing over all such cylinders, we get

$$
\sum_{C \in \mathcal{U}_{\varepsilon}}|C|_{\lambda}^{t}=\sum_{C \in \mathcal{U}_{\varepsilon}}|C|_{\gamma}^{u} e^{u \eta n(C)}\left(\frac{\lambda^{t}}{\gamma^{u}}\right)^{\chi_{n}(C)-2} .
$$

By definition of $\Omega_{\gamma}^{\prime \prime}$, we have $\chi_{n}(C) \leqslant 10 \operatorname{Dr}(\gamma) n$ and thus $\left(\frac{\lambda^{t}}{\gamma^{u}}\right)^{\chi_{n}(C)} \geqslant e^{-u \eta n}$ provided $\gamma$ is sufficiently close to $\frac{1}{2}$. Therefore the right hand side in the formula diverges as $\gamma \searrow \frac{1}{2}$ and $\varepsilon \rightarrow 0$. Furthermore, the mesh size of $\mathcal{U}_{\varepsilon}$ is different for parameter $\lambda$ and $\gamma$, but they tend to 0 for both parameters simultaneously as $\varepsilon \rightarrow 0$. Therefore 
$t \leqslant \operatorname{dim}_{H}\left(\Omega_{\lambda}\right)$. Taking the limits $\gamma \searrow \frac{1}{2}, u \nearrow 1$ and $\eta \rightarrow 0$, we obtain the required lower bound $\operatorname{dim}_{H}\left(\Omega_{\lambda}\right) \geqslant \frac{-\log 4}{\log \lambda(1-\lambda)}$.

For the upper bound, we take $u>1$ and $\eta=0$. Then the $u$-dimensional Hausdorff mass of $\varepsilon$-covers $\mathcal{U}_{\varepsilon}$ converges for parameter $\gamma=\frac{1}{2}$. So now, taking $\eta=0$ and the limit $u \searrow 1$, gives the same upper bound $\operatorname{dim}_{H}\left(\Omega_{\lambda}\right) \leqslant \frac{-\log 4}{\log \lambda(1-\lambda)}$. This completes the proof for $\lambda<\frac{1}{2}$. Finally, monotonicity of $\lambda \mapsto \operatorname{dim}_{H}\left(\Omega_{\lambda}\right)$ gives $\operatorname{dim}_{H}\left(\Omega_{\lambda}\right)=1$ for $\lambda=\frac{1}{2}$.

Remark 4. Notice the striking symmetry: $\operatorname{dim}_{\text {hyp }}\left(F_{\lambda}\right)=\operatorname{dim}_{H}\left(\Omega_{1-\lambda}\right)$. We can explain this using an argument from [SV], namely a coding of the system $\left((0,1], F_{\lambda}\right)$ based on " $\lambda$-adic" partitions that are defined inductively by starting with $[0,1]$, and dividing all intervals of the previous stage into two parts of relative lengths $1-\lambda$ (with symbol 0 ) and $\lambda$ (with symbol 1$)$. This means that Lebesgue measure on $[0,1]$ corresponds to $(1-\lambda, \lambda)$ Bernoulli measure on $\{0,1\}^{\mathbb{N}_{0}}$.

As a result, if $x$ has code $0^{n_{0}} 10^{n_{1}} 10^{n_{2}} 10^{n_{3}} 1 \ldots$ then $x \in W_{n_{1}}$,

$$
F_{\lambda}(x) \in \begin{cases}W_{n_{0}+n_{1}-1} & \text { if } x \notin W_{1} \\ W_{n_{1}} & \text { if } x \in W_{1} .\end{cases}
$$

and in general,

$$
F_{\lambda}^{k}(x) \in \begin{cases}W_{j+n_{k}-1} & \text { if } F_{\lambda}^{k-1}(x) \in W_{j}, j \geqslant 2 \\ W_{n_{k}} & \text { if } F_{\lambda}^{k-1}(x) \in W_{1} .\end{cases}
$$

Let $Y$ be the set of point such that 0 s dominate in their codes, i.e.,

$$
Y=\left\{y \in[0,1]: \lim _{r \rightarrow \infty} \sum_{j=0}^{r-1} n_{j}(y)-r \rightarrow \infty\right\} .
$$

These are precisely the points such that $F_{\lambda}^{r}(y) \rightarrow 0$, and in fact $F_{\lambda}^{r}(y) \in \cup_{i \geqslant j} W_{i}$ if $j=\sum_{j=0}^{r-1} n_{j}-r$ with equality if $F_{\lambda}^{j}(y)$ does not linger in $W_{0}$ for successive iterates $j \leqslant r$. Stratmann 8 Vogt show that $\operatorname{dim}_{H}(Y)$ is given by (23).

Let us now form $\hat{Y}$ as the set of points $\hat{y}$ with opposite codes as $Y$, i.e., $\hat{y}$ is the point obtained by changing all $0 s$ in the code of $y$ by 1 s and vice versa, and let us also change $\lambda$ to $\hat{\lambda}=1-\lambda$. Then $\operatorname{dim}_{H}\left(\hat{Y}_{\hat{\lambda}}\right)=\operatorname{dim}_{H}\left(Y_{\lambda}\right)$. But $\hat{Y}$ are points in whose code 1 s dominate, so their orbits visit only finitely many intervals $W_{j}$, and hence $\operatorname{dim}\left(\hat{Y}_{\hat{\lambda}}\right)=\operatorname{dim}_{\text {hyp }}\left(F_{\hat{\lambda}}\right)$, which explains the symmetry.

The only exception for this argument are point $\hat{y}$ that remain in $W_{0}$ for a long time $n_{k}$ ( $a$ block of $n_{k}$ ones in the code) and then visit $W_{j_{k}}$ for $1 \ll j_{k} \ll n_{k}$ (a block of $j_{k}$ zeroes in the code). The regularity of such codes makes is plausible that the Hausdorff dimension of such points is small and hence has no effect on the equality $\operatorname{dim}\left(\hat{Y}_{\hat{\lambda}}\right)=\operatorname{dim}_{\text {hyp }}\left(F_{\hat{\lambda}}\right)$.

\section{Topological and Gurevich Pressure}

In this section we present the classical definition (see [R1, W1, W2]) of topological pressure along with a Gurevich definition of pressure for countable Markov graphs 
which allows us to prove Theorem B and Corollary 1 . The results here also set the stage for the proof of the null recurrent case in Section 7.

Let $f: X \rightarrow X$ be a continuous map on a metric space. Following Bowen [Bo], let

$$
d_{n}(x, y):=\max \left\{d\left(f^{k}(x), f^{k}(y)\right): 0 \leqslant k<n\right\}
$$

Given $\varepsilon>0$ we say that a finite set $E \subset X$ is $(n, \varepsilon)$-separated if $d_{n}(x, y)>\varepsilon$ for every $x, y \in E$ such that $x \neq y$. Bowen showed that topological entropy coincides with the exponential growth rate in $n$ of the maximal cardinality of $(n, \varepsilon)$-separated sets, but in order to obtain pressure, one needs to compute ergodic sums of of the potential on each point in an $(n, \varepsilon)$-separated set. Let $E_{n, \varepsilon}$ be the collection of all $(n, \varepsilon)$-separated sets. Define

$$
\Gamma_{n, \varepsilon}(\phi):=\sup _{E_{n, \varepsilon}} \sum_{x \in E_{n, \varepsilon}} e^{S_{n} \phi(x)}
$$

where $S_{n} \phi(x):=\phi(x)+\cdots+\phi \circ \sigma^{n-1}(x)$. The classical topological pressure introduced in [R1, W1] is

$$
P_{\text {top }}(\phi):=\lim _{\varepsilon \rightarrow 0} \limsup _{n \rightarrow \infty} \frac{1}{n} \log \Gamma_{n, \varepsilon} .
$$

Of course, our maps $F_{\lambda}:(0,1] \rightarrow(0,1]$ are not continuous as interval maps. However, we can still compute topological pressure for them.

Remark 5. In the compact setting, since all metrics generating the same topology are uniformly equivalent ( $d_{1}$ and $d_{2}$ are called uniformly equivalent if the identity maps from $\left(X, d_{1}\right)$ to $\left(X, d_{2}\right)$ and vice versa are both uniformly continuous), the value of the pressure does not depend upon the metric (see [W2, Section 7.2]). However, in noncompact settings this may no longer be the case. This is one of the motivations for the alternative notion of pressure given in the next subsection, which in our situation is shown to agree with $P_{\text {top }}(\phi)$.

Since $F_{\lambda}$ preserves the countable Markov partition $\left\{W_{k}\right\}_{k \in \mathbb{N}}$ it is natural to use a countable Markov shift (CMS) on alphabet $\mathbb{N}$. By the definition of $W_{n}$ as half-open intervals, every point (rather than almost every) has a well-defined symbolic itinerary, no information is lost when passing from the interval to symbolic dynamics. With the theory we present here we can interpret some of the results proved above about eigenvalues of matrices in terms of the pressure.

Let $\sigma: \Sigma \rightarrow \Sigma$ be a one-sided Markov shift with a countable alphabet $\mathbb{N}$. That is, there exists a matrix $\left(t_{i j}\right)_{\mathbb{N} \times \mathbb{N}}$ of zeros and ones (with no row and no column made entirely of zeros) such that

$$
\Sigma=\left\{x \in \mathbb{N}^{\mathbb{N}_{0}}: t_{x_{i} x_{i+1}}=1 \text { for every } i \in \mathbb{N}_{0}\right\},
$$

and the shift map is defined by $\sigma\left(x_{0} x_{1} \cdots\right)=\left(x_{1} x_{2} \cdots\right)$. We say that $(\Sigma, \sigma)$ is a countable Markov shift. We equip $\Sigma$ with the topology generated by the cylinder sets

$$
\left[e_{0} \cdots e_{n-1}\right]=\left\{x \in \Sigma: x_{j}=e_{j} \text { for } 0 \leqslant j<n\right\} .
$$

By making the move from the interval to the coding space $\Sigma$ we lose connectedness, but gain smoothness for our potentials. 
Given a function $\phi: \Sigma \rightarrow \mathbb{R}$, for each $n \geqslant 1$ we define the variation on $n$-cylinders

$$
V_{n}(\phi)=\sup \left\{|\phi(x)-\phi(y)|: x, y \in \Sigma, x_{i}=y_{i} \text { for } 0 \leqslant i<n\right\} .
$$

We say that $\phi$ has summable variations if $\sum_{n=2}^{\infty} V_{n}(\phi)<\infty$. We will sometimes refer to $\sum_{n=2}^{\infty} V_{n}(\phi)$ as the distortion bound for $\phi$. Clearly, if $\phi$ has summable variations then it is continuous. We say that $\phi$ is weakly Hölder continuous if $V_{n}(\phi)$ decays exponentially. If this is the case then it has summable variations. In what follows we assume $(\Sigma, \sigma)$ to be topologically mixing (see [S1, Section 2] for a precise definition).

Based on work of Gurevich [G1, G2], Sarig [S1] introduced a notion of pressure for countable Markov shifts which does not depend upon the metric of the space and which satisfies a Variational Principle. Let $(\Sigma, \sigma)$ be a topologically mixing countable Markov shift, fix a symbol $e_{0}$ in the alphabet $S$ and let $\phi: \Sigma \rightarrow \mathbb{R}$ be a potential of summable variations. We let the local partition function at $\left[e_{0}\right]$ be

$$
Z_{n}\left(\phi,\left[e_{0}\right]\right):=\sum_{x: \sigma^{n} x=x} e^{S_{n} \phi(x)} \chi_{\left[e_{0}\right]}(x)
$$

and

$$
Z_{n}^{*}\left(\phi,\left[e_{0}\right]\right):=\sum_{\substack{x: \sigma^{n} x=x, x: \sigma^{k} x \notin\left[e_{0}\right] \text { for } 0<k<n}} e^{S_{n} \phi(x)} \chi_{\left[e_{0}\right]}(x),
$$

where $\chi_{\left[e_{0}\right]}$ is the characteristic function of the 1-cylinder $\left[e_{0}\right] \subset \Sigma$, and $S_{n} \phi(x)$ is $\phi(x)+$ $\cdots+\phi \circ \sigma^{n-1}(x)$. The so-called Gurevich pressure of $\phi$ is defined by the exponential growth rate

$$
P_{G}(\phi):=\lim _{n \rightarrow \infty} \frac{1}{n} \log Z_{n}\left(\phi,\left[e_{0}\right]\right) .
$$

Since $\sigma$ is topologically mixing, one can show that $P_{G}(\phi)$ does not depend on $e_{0}$. If $(\Sigma, \sigma)$ is the full-shift on a countable alphabet then the Gurevich pressure coincides with the notion of pressure introduced by Mauldin \& Urbański [MU1.

We defined transience/recurrence of a system in the introduction in terms of the relevant measures there. In the CMS context, as proved in [S2], these are equivalent to the following definitions. The potential $\phi$ is said to be recurrent if

$$
\sum_{n} e^{-n P_{G}(\phi)} Z_{n}(\phi)=\infty .
$$

Otherwise $\phi$ is transient. Moreover, $\phi$ is called positive recurrent if it is recurrent and

$$
\sum_{n} n e^{-n P_{G}(\phi)} Z_{n}^{*}(\phi)<\infty .
$$

The following can be shown using the proof of [S1, Theorem 3].

Proposition 4 (Variational Principle). If $(\Sigma, \sigma)$ is topologically mixing and $\phi: \Sigma \rightarrow \mathbb{R}$ has summable variations, $\phi<\infty$ and $\phi$ is weakly Hölder continuous, then

$$
P_{G}(\phi)=P(\phi) \text {. }
$$

\footnotetext{
${ }^{1}$ The convergence of this series is independent of the cylinder set $\left[e_{0}\right]$, so we suppress it in the notation.
} 
Let us stress that $P(\phi)$ only depends on the Borel structure of the space and not on the metric . Therefore, $P_{G}(\phi)$ must also be independent of the metric on the space.

The Gurevich pressure also has the property that it can be approximated by its restriction to compact sets. More precisely [S1, Corollary 1]:

Proposition 5 (Approximation Property). If $(\Sigma, \sigma)$ is topologically mixing and $\phi$ : $\Sigma \rightarrow \mathbb{R}$ is weakly Hölder continuous then

$$
P_{G}(\phi)=\sup \left\{P_{t o p \mid K}(\phi): \emptyset \neq K \subset \Sigma, K \text { is compact and shift-invariant }\right\},
$$

where $P_{t o p \mid K}(\phi)$ is the topological pressure on $K$.

At this point we can prove that for our systems, $P_{\text {top }}$ and $P_{G}$ coincide. This result is similar to [S1, Proposition 1.3]

Proposition 6. For $\lambda \in(0,1)$ and $t \geqslant 0, P_{t o p}\left(-t \log \left|F_{\lambda}^{\prime}\right|\right)=P_{G}\left(-t \log \left|F_{\lambda}^{\prime}\right|\right)$.

Proof. For a subset $K \subset X$, let $\left.\Gamma_{n, \varepsilon}(\phi)\right|_{K}$ be the above quantity restricted to the set $K$. We first claim that for our map $F$ and for each $\varepsilon>0$, there exists $K_{\varepsilon} \subset I$ such that

$$
\Gamma_{n, \varepsilon} \leqslant\left. n \Gamma_{n, \varepsilon}\right|_{K_{\varepsilon}} .
$$

Indeed, for $\varepsilon>0$ there exists a minimal $n(\varepsilon) \geqslant 1$ such that $\left|\cup_{n \geqslant n(\varepsilon)} A_{n}\right|<\varepsilon$. Set $K_{\varepsilon}:=I \backslash\left(\cup_{n \geqslant n(\varepsilon)} A_{n}\right)$. Then by the structure of $F$, there is just one element from $I \backslash K_{\varepsilon}$ entering $K_{\varepsilon}$ at each successive iterate of $F$. These new contributions (which are initially of weight $(\lambda(1-\lambda))^{n t}$, but eventually can be of the form $\left.(1-\lambda)^{n t} \lambda^{k}\right)$ can be paired with a summand already in the sum for $\left.\Gamma_{n}\right|_{K_{\varepsilon}}$. The number of these new terms generated up to time $n$ is $\leqslant n$ so (27) is in fact a big over-estimate.

The Variational Principle for finite shifts on compact sets implies $P_{\text {top }}\left(-t \log \left|F^{\prime}\right|_{K_{\varepsilon}} \mid\right)=$ $P_{G}\left(-t \log \left|F^{\prime}\right|_{K_{\varepsilon}} \mid\right)$. Since (27) implies

$$
\lim _{\varepsilon \rightarrow 0} P_{G}\left(-t \log \left|F^{\prime}\right|_{K_{\varepsilon}} \mid\right) \rightarrow P_{\text {top }}\left(-t \log \left|F^{\prime}\right|\right),
$$

adding this to Proposition 5 gives $P_{\text {top }}\left(-t \log \left|F^{\prime}\right|\right)=P_{G}\left(-t \log \left|F^{\prime}\right|\right)$, as required.

We use the standard transfer operator $\left(L_{\phi} v\right)(x)=\sum_{\sigma y=x} e^{\phi(y)} v(y)$, with dual operator $L_{\phi}^{*}$. Notice that a measure $m$ is $\phi$-conformal if and only if $L_{\phi}^{*} m=m$.

The following theorem is $\underline{\mathrm{S} 2}$, Theorem 1].

Theorem 4. Suppose that $(\Sigma, \sigma)$ is topologically mixing, $\phi: \Sigma \rightarrow \mathbb{R}$ has summable variations and $P_{G}(\phi)<\infty$. Then $\phi$ is recurrent if and only if there exists $\lambda>0$ and a conservative sigma-finite measure $m_{\phi}$ finite and positive on cylinders, and a positive continuous function $h_{\phi}$ such that $L_{\phi}^{*} m_{\phi}=\lambda m_{\phi}$ and $L_{\phi} h_{\phi}=\lambda h_{\phi}$. In this case $\lambda=e^{P_{G}(\phi)}$. Moreover,

(1) if $\phi$ is positive recurrent then $\int h_{\phi} d m_{\phi}<\infty$;

(2) if $\phi$ is null recurrent then $\int h_{\phi} d m_{\phi}=\infty$.

Moreover the next theorem follows by [S2, Theorem 2]: 
Theorem 5. Suppose that $(\Sigma, \sigma)$ is topologically mixing and $\phi: \Sigma \rightarrow \mathbb{R}$ is weakly Hölder continuous and positive recurrent. Then for the measure $d \mu=h_{\phi} d m_{\phi}$ given by Theorem 4, if $-\int \phi d \mu$ is finite then $\mu$ is an equilibrium state for $\phi$.

Proposition 7. Suppose that $(\Sigma, \sigma)$ is topologically mixing and $\phi: \Sigma \rightarrow \mathbb{R}$ is Hölder continuous, has finite Gurevich pressure and is transient or null recurrent. Then there is no equilibrium state for $\phi$.

Proof. We may assume that $\phi$ has $P(\phi)=0$, otherwise we can shift by $P(\phi)$. Now we use an inducing argument. We fix a state $a \in S$, and derive the induced system $(\bar{X}, \bar{\sigma}, \bar{\phi})$ as a first return map to $a$. This is the full shift on countably many symbols, which, as shown in MU2 and S4 has many strong properties. We will use these to guarantee that we have no equilibrium state in the non-positive recurrent case.

We begin by noting that [MU2] and [S4], since $(\bar{X}, \bar{\sigma})$ is the full shift, $\bar{\phi}$ is necessarily positive recurrent whenever $P(\bar{\phi})<\infty$ for any choice of $a \in S$. We can in fact show that in all cases $P(\bar{\phi}) \leqslant 0$. Indeed, if $P(\bar{\phi})>0$ then by Proposition 5 we can take a compact invariant subset of $(\bar{X}, \bar{\phi})$ which still has strictly positive pressure and a corresponding equilibrium state $\bar{\nu}$. By the Abramov formula for the projection $\nu$ of $\bar{\nu}$ to $\Sigma$, we have $h(\nu)+\int \phi d \nu>0$ contradicting Proposition 4.

Now if there is an equilibrium state $\mu$ (hence with unit mass) for $\phi$ then $h(\mu)+\int \phi d \mu=$ 0 . Let $a$ be a state which is given positive mass by $\mu$ and let $\bar{\mu}$ be the rescaled measure on $[a]$. Then the Abramov formula implies that $h(\bar{\mu})+\int \bar{\phi} d \bar{\mu}=0$ and so Proposition 4 implies that $P(\bar{\phi}) \geqslant 0$. Thus $P(\bar{\phi})=0$. We now apply [S1, Corollary 2], which when added to part (2) of [S4, Corollary 2], says that any equilibrium state for $\bar{\phi}$ must be of the form obtained in Theorem 5. Thus $d \bar{\mu}=h_{\bar{\phi}} d m_{\bar{\phi}}$ where $L_{\bar{\phi}} h_{\bar{\phi}}=h_{\bar{\phi}}$ and $L_{\bar{\phi}}^{*} m_{\bar{\phi}}=m_{\bar{\phi}}$. The functional form of the Kac's Lemma, shown in [S3, Lemma 3] implies that $\mu$ must also be of this form (i.e., $d \mu=h_{\phi} d m_{\phi}$ where $L_{\phi} h_{\phi}=h_{\phi}$ and $L_{\phi}^{*} m_{\phi}=m_{\phi}$ ), which by Theorem 4 contradicts the assumption that $\mu$ was not positive recurrent.

We can now use the theory for thermodynamic formalism for countable Markov shifts to prove Theorem $\mathrm{B}$ and Corollary 1 . These follow almost immediately from the results presented in this and in previous sections since, as shown below, the transfer operator can be interpreted in terms of the matrix $B^{t}$.

Proof of Theorem B. We start by clarifying the link between transfer operators for simple potentials and their matrix representations. Let $(\Sigma, \sigma)$ be a CMS where, for simplicity, we take $\Sigma=\mathbb{N}^{\mathbb{N}}$. Then given a potential $\phi: \Sigma \rightarrow \mathbb{R}$ which only depends on one coordinate $\left(e . g . V_{1}(\phi)=0\right)$, one can form the corresponding infinite matrix $D=D_{\phi}=\left(d_{i, j}\right)_{i, j \in \mathbb{N}}$ as $d_{i, j}=\phi(i)$ for all $i \in \mathbb{N}$. Now for a function $\xi: \Sigma \rightarrow \mathbb{R}$ which depends only on one coordinate, we define $\underline{\xi}$ to be the vector $(\xi(1), \xi(2), \ldots)$, and $\underline{e}_{i}$ to be the row vector with all zeros except in the $i$-th entry, which is 1 . Then we can compute that for any $x$ in the 1-cylinder $[i]$,

$$
\left(L_{\phi} \xi\right)(x)=\underline{e}_{i}(\underline{\xi} \cdot D) \text { and }\left(L_{\phi}^{*} \xi\right)([i])=\left(D \cdot \underline{\xi}^{T}\right) \underline{e}_{i} .
$$

Thus the leading eigenvalue of the matrix $D$ is the exponential of the Gurevich pressure of $\phi$. 
The fact that the leading eigenvalue of $B^{t}$ is the exponential of the pressure follows from (28) and thus the expression for $P\left(\Phi_{t}\right)$ follows from Theorem 3 . The fact that the pressure function is not $C^{2}$ at $t_{0}=\frac{-\log 2}{\log \lambda}$ follows since by Lemma 1, $D^{2} \psi\left(t_{0}\right)>0$.

The existence of $\mu_{t}$ when $\lambda^{t}<1 / 2$ follows from Proposition 1, Uniqueness follows from Theorem 4. The fact that $\mu_{t}$ is an equilibrium state follows from Theorem 5, The non-existence of an equilibrium state when $\lambda^{t} \geqslant 1 / 2$ follows from Proposition 7 ,

We finish this section with the proof of Corollary 1 .

Proof of Corollary 1, Let us recall equation (3):

$$
\begin{aligned}
P\left(\Phi_{t}\right) & =P_{G}\left(\Phi_{t}\right)=P_{\text {top }}\left(\Phi_{t}\right)=P_{\text {Conf }}\left(\Phi_{t}\right)=\log \sigma\left(B^{t}\right) \\
& =\lim _{K \rightarrow \infty} \log \sigma\left(B_{K}^{t}\right)=\lim _{K \rightarrow \infty} \log \sigma\left(A_{K}^{t}\right) .
\end{aligned}
$$

The first equality follows by Proposition 4. The second follows by Proposition 6. The third and fourth follow by Theorem 4 . The fifth follows by Proposition 5 and the sixth follows by Lemma [2. If $t=0$, Theorem 3 gives $\lim _{K \rightarrow \infty} \log \sigma\left(B_{K}\right)=$ $\lim _{K \rightarrow \infty} \log \sigma\left(A_{K}\right)=\log 4$.

\section{Null RECURRENT CASE}

Lemma 3. If $\lambda^{t}=1 / 2$ then $\left((0,1], F_{\lambda}, \Phi_{t}\right)$ is null recurrent.

Proof. Since $Z_{k}\left(\Phi_{t}, A_{1}\right)=\underline{1} \cdot D^{k-1} \cdot(1,0,0, \ldots)^{T}$ for

$$
D:=\left(\begin{array}{ccccccc}
\frac{1}{2} & \frac{1}{2} & \frac{1}{2} & \frac{1}{2} & \frac{1}{2} & \ldots & \ldots \\
\frac{1}{4} & \frac{1}{4} & \frac{1}{4} & \frac{1}{4} & \frac{1}{4} & \ldots & \ldots \\
0 & \frac{1}{4} & \frac{1}{4} & \frac{1}{4} & \frac{1}{4} & \frac{1}{4} & \ldots \\
0 & 0 & \frac{1}{4} & \frac{1}{4} & \frac{1}{4} & \frac{1}{4} & \ldots \\
\vdots & \vdots & 0 & \frac{1}{4} & \frac{1}{4} & \frac{1}{4} & \ldots \\
\vdots & \vdots & \vdots & \vdots & \vdots & \vdots & \ddots
\end{array}\right),
$$

the lemma can be proved by determining the form of the first column of the matrices $D^{n-1}$ (although, of course, we only really care about the term in the top left corner). Note that the leading eigenvalue of this matrix is 1 , so a priori, the terms of interest could decrease at any subexponential rate.

Claim 2. For $k \geqslant 1$, we have

$$
\left(D^{k}\right)_{i, 1}= \begin{cases}p_{k, k-i+1} / 2^{2 k-1} & \text { if } i=1 \\ p_{k, k-i+2} / 2^{2 k} & \text { if } 2 \leqslant i \leqslant k+1 \\ 0 & \text { if } i>k+1\end{cases}
$$

for binomial coefficients $p_{k, i}:=\left(\begin{array}{c}k+2(i-1) \\ 2(i-1)\end{array}\right)$. 
Proof. We denote the first column of $D^{n}$ by $\underline{v}^{n}$. The columns $\underline{v}^{1}, \ldots, \underline{v}^{5}$ are:

$$
\left(\begin{array}{c}
\frac{1}{2} \\
\frac{1}{2^{2}} \\
0 \\
0 \\
0 \\
0 \\
0 \\
\vdots
\end{array}\right),\left(\begin{array}{c}
\frac{3}{2^{3}} \\
\frac{3}{2^{4}} \\
\frac{1}{2^{4}} \\
0 \\
0 \\
0 \\
0 \\
\vdots
\end{array}\right),\left(\begin{array}{c}
\frac{10}{2^{5}} \\
\frac{10}{2^{6}} \\
\frac{4}{2^{6}} \\
\frac{1}{2^{6}} \\
0 \\
0 \\
0 \\
\vdots
\end{array}\right),\left(\begin{array}{c}
\frac{35}{2^{7}} \\
\frac{35}{2^{8}} \\
\frac{15}{2^{8}} \\
\frac{5}{2^{8}} \\
\frac{1}{2^{8}} \\
0 \\
0 \\
\vdots
\end{array}\right),\left(\begin{array}{c}
\frac{126}{2^{9}} \\
\frac{126}{2^{10}} \\
\frac{56}{2^{10}} \\
\frac{21}{2^{10}} \\
\frac{6}{2^{10}} \\
\frac{1}{2^{10}} \\
0 \\
\vdots
\end{array}\right) .
$$

Let us denote the numerator of the $i$-th entry of $\underline{v}^{k}$ by $n_{k, i}$. We obtain the following relations:

$$
n_{k, i}= \begin{cases}2 n_{k-1,1}+n_{k-1,2}+n_{k-1,3}+\cdots+n_{k-1, n} & \text { for } i=1,2, \\ n_{k-1, i-1}+n_{k-1,2}+n_{k-1,3}+\cdots+n_{k-1, n} & \text { for } 3 \leqslant i \leqslant k+1 .\end{cases}
$$

Clearly the denominator is $2^{2 k-1}$ for $i=1$ and $2^{2 k}$ for $0 \leqslant i \leqslant k+1$. The claim follows by the observation that the formula for $p_{k, k-i+2}$ is the same as that for $n_{k, i}$.

Note that another way to prove this is by examining the recursive relations in Pascal's triangle - the terms $p_{k, k-i+2}$ can be observed on the $(k+1)$-st diagonal.

Stirling's formula gives $p_{k, k} \approx(1 / 2) 2^{2 k} / \sqrt{2 \pi k}$. Therefore, the claim implies that $Z_{k}\left(\Phi_{t}, A_{1}\right) \geqslant C / \sqrt{k}$ for some $C>0$, and so the system is indeed recurrent.

To prove null recurrence, we appeal to Theorem 4. Given $\rho_{t}$ the eigenfunction for $L_{\Phi_{t}}$ and $m_{t}$, the $\left(t, P_{\text {Conf }}\left(\Phi_{t}\right)\right)$-conformal measure, it suffices to show that $\int \rho_{t} d m_{t}=\infty$.

These have been computed earlier and combine to give $\int_{A_{i}} \rho_{t} d m_{t}=\left(\frac{\lambda^{t}}{1-\lambda^{t}}\right)^{i-1}$. (Note we can rescale $m_{t}$ and $\rho_{t}$, but not in a way which would change our result.) Since in this case $\lambda^{t}=1 / 2$, we obtain $\int \rho_{t} d m_{t}=\infty$ as required.

\section{REFERENCES}

[Aa] J. Aaronson, An introduction to infinite ergodic theory, Mathematical Surveys and Monographs, 50, American Mathematical Society, Providence, RI, 1997.

[BS] J. Bobok, M. Soukenka, On piecewise affine interval maps with countably many laps, Preprint 2010.

[Bo] R. Bowen, Entropy for group endomorphisms and homogeneous spaces, Trans. Amer. Math. Soc. 153 (1971) 401-414.

[BKNS] H. Bruin, G. Keller, T. Nowicki, S. van Strien, Wild Cantor attractors exist, Ann. of Math. 143 (1996) 97-130.

[G1] B. M. Gurevič, Topological entropy for denumerable Markov chains, Dokl. Akad. Nauk SSSR 10 (1969) 911-915.

[G2] B. M. Gurevič, Shift entropy and Markov measures in the path space of a denumerable graph, Dokl. Akad. Nauk SSSR 11 (1970) 744-747.

[IT] G. Iommi, M. Todd, Transience in dynamical systems, Ergodic Theory Dynam. Systems 33 (2013) 1450-1476.

[LY] A. Lasota, J. Yorke, On the existence of invariant measures for piecewise monotonic transformations, Trans. Amer. Math. Soc. 186 (1973) 481-488. 
[MU1] R. Mauldin, M. Urbański, Dimensions and measures in infinite iterated function systems, Proc. London Math. Soc. (3) 73 (1996) 105-154.

[MU2] R. Mauldin, M. Urbański, Gibbs states on the symbolic space over an infinite alphabet, Israel J. Math. 125 (2001) 93-130.

[MR] M. Misiurewicz, P. Raith, Strict inequalities of the entropies of transitive piecewise monotone maps, Discrete Contin. Dyn. Syst. 13 (2003), 451-468.

[MS] C. G. Moreira, D. Smania, Metric stability for random walks (with applications in renormalization theory), Preprint, 2005 (updated 2009), arXiv:math/0503736

[PRS] F. Przytycki, J. Rivera-Letelier, S. Smirnov, Equality of pressures for rational functions, Ergodic Theory Dynam. Systems 24 (2004) 891-914.

[R1] D. Ruelle, Statistical mechanics on a compact set with $Z^{\nu}$ action satisfying expansiveness and specification, Trans. Amer. Math. Soc. 187 (1973) 237-251.

[R2] D. Ruelle, Thermodynamic Formalism, Encyclopedia of Mathematics and its Applications, 5. Addison-Wesley, Reading, MA, 1978.

[Rt] S. Ruette, Mixing $C^{r}$ maps of the interval without maximal measure, Israel J. Math. 127 (2002), 253-277.

[Sl] I. Salama, Topological entropy and recurrence of countable chains, Pacific J. Math. 134 (1988) $325-341$.

[S1] O. Sarig, Thermodynamic formalism for countable Markov shifts, Ergodic Theory Dynam. Systems 19 (1999) 1565-1593.

[S2] O. Sarig, Thermodynamic formalism for null recurrent potentials, Israel J. Math. 121 (2001) 285-311.

[S3] O. Sarig, Phase transitions for countable Markov shifts, Comm. Math. Phys. 217 (2001) $555-577$.

[S4] O. Sarig, Existence of Gibbs measures for countable Markov shifts, Proc. Amer. Math. Soc. 131 (2003) 1751-1758.

[S5] O. Sarig, Lectures Notes on Thermodynamic Formalism for Topological Markov Shifts (2009).

[SS] J. Schmeling, B. Stratmann, The Hausdorff dimension of the set of dissipative points for a Cantor-like model set for singly cusped parabolic dynamics, Kodai Mathematical Journal 32 (2009) 179-196.

[SV] B. Stratmann, R. Vogt, Fractal dimension of dissipative sets, Nonlinearity 10 (1997) 565-577.

[W1] P. Walters, A variational principle for the pressure of continuous transformations, Amer. J. Math. 97 (1975) 937-971.

[W2] P. Walters, An Introduction to Ergodic Theory, Graduate Texts in Mathematics 79, Springer, 1981.

Department of Mathematics, University of Surrey, Guildford, Surrey, GU2 7XH, UK

E-mail address: h.bruin@surrey.ac.uk

$U R L:$ http://personal.maths.surrey.ac.uk/st/H.Bruin/

Mathematical Institute, University of St Andrews, North Haugh, St Andrews, Fife, KY16 9SS, SCOTLAND

E-mail address: mjt20@st-andrews.ac.uk

$U R L:$ http://www.mcs.st-and.ac.uk/ miket/index.html 